The AstrophysicAl Journal, 530:508-524, 2000 February 10

(c) 2000. The American Astronomical Society. All rights reserved. Printed in U.S.A.

\title{
HIGH-ORDER UPWIND SCHEMES FOR MULTIDIMENSIONAL MAGNETOHYDRODYNAMICS
}

\author{
P. LONDRILLO \\ Osservatorio Astronomico, Via Ranzani 1, 40127 Bologna, Italy; londrillo@bo.astro.it \\ AND \\ L. DEL ZANNA \\ Dipartimento di Astronomia e Scienza dello Spazio, Largo Enrico Fermi 5, 50125, Firenze, Italy; 1dz@arcetri.astro.it \\ Received 1999 April 30; accepted 1999 September 29
}

\begin{abstract}
A general method for constructing high-order upwind schemes for multidimensional magnetohydrodynamics (MHD), having as a main built-in condition the divergence-free constraint $\boldsymbol{\nabla} \cdot \boldsymbol{B}=0$ for the magnetic field vector $\boldsymbol{B}$, is proposed. The suggested procedure is based on consistency arguments, by taking into account the specific operator structure of MHD equations with respect to the reference Euler equations of gasdynamics. This approach leads in a natural way to a staggered representation of the $\boldsymbol{B}$ field numerical data in which the divergence-free condition in the cell-averaged form, corresponding to second-order accurate numerical derivatives, is exactly fulfilled. To extend this property to higher order schemes, we then give general prescriptions to satisfy a $(r+1)$ th order accurate $\boldsymbol{\nabla} \cdot \boldsymbol{B}=0$ relation for any numerical $\boldsymbol{B}$ field having a $\boldsymbol{r}$ th order interpolation accuracy. Consistency arguments lead also to a proper formulation of the upwind procedures needed to integrate the induction equations, assuring the exact conservation in time of the divergence-free condition and the related continuity properties for the $\boldsymbol{B}$ vector components. As an application, a third-order code to simulate multidimensional MHD flows of astrophysical interest is developed using essentially nonoscillatory-based reconstruction algorithms. Several test problems to illustrate and validate the proposed approach are finally presented.
\end{abstract}

Subject heading: methods: numerical — MHD

\section{INTRODUCTION}

Many astrophysical plasmas, such as stellar (or galactic) atmospheres and winds, accretion disks, and jets, can be described by the set of compressible magnetohydrodynamic (MHD) equations with dissipative terms neglected, since kinetic effects of astrophysical plasmas are quite small on dominant macroscopic scales. In these physical regimes, dynamical effects give rise to complex time-dependent flows in which localized sharp modes like shocks and current sheets couple with distributed nonlinear waves. It is therefore a main challenge to computational astrophysics to take properly into account both dynamical components.

Centered finite differences or spectral schemes are well suited for smooth fields and can support discontinuities only by introducing enough viscous/resistive dissipation. In this way field discontinuities are represented with poor resolution, and artificial heating takes place. On the other hand, upwind schemes achieve shock capturing and localized high resolution in a natural way. When discontinuous solutions are of main interest, second-order (in time and space) schemes are usually adopted, since they reconcile resolution with efficiency and stability needs. However, in the general case, in which coherent sharp field structures are embedded in a turbulent background, second-order accuracy is no longer the optimal one, since the (implicit) numerical viscosity is still too high to resolve properly small-scale motions. There are therefore compelling computational and physical reasons to develop higher order upwind schemes for MHD flows.

In recent years progress has been made in extending Godunov-type schemes developed for the Euler system of gasdynamics to MHD, with main emphasis on the wave characteristic structure. In Brio \& Wu (1988), and Roe \& Balsara (1996), the problem of nonstrict hyperbolicity of the
MHD system has been addressed by introducing proper regularity factors to renormalize the eigenvectors and assure their linear independence. The related problem of constructing the Roe linearized matrix for MHD case has also been solved (Cargo \& Gallice 1997; Balsara 1998a). Based on these achievements, second-order upwind codes using either Godunov's or Roe's method have then been constructed and tested, mainly for one-dimensional MHD problems (e.g., Ryu \& Jones 1995; Zachary, Malagoli, \& Colella 1994; Balsara 1998b; Dai \& Woodward 1998, and references therein).

Specific new problems and limitations have to be considered in going to higher order and multidimensional MHD case. Upwind schemes are usually constructed by first projecting fluid variables at each grid point on the space of characteristic variables. The decomposition procedure allows to achieve a better resolution since interacting discontinuities of fluid variables become uncoupled in the space of characteristic variables. This technique is usually adopted in existing MHD codes, too, but there is no clear evidence that it can work even for higher order schemes (Barmin, Kulikovskiy, \& Pogorelov 1996). Moreover, the computational cost of projecting field variables onto the MHD seven-component characteristic space may become prohibitive when moving to higher order and higher dimensional schemes. Therefore, as already experienced in the context of numerical gasdynamics, a search for high-order shock-capturing schemes in which no characteristic decomposition is needed and in which attention is shifted more to a vanishing viscosity entropy satisfying model equations, rather than on approximate Riemann solvers, appears to be more promising.

A second important issue in multidimensional MHD schemes comes from the need to satisfy the divergence-free 
condition of the magnetic field vector. This property is a crucial one for two main reasons (Balsara \& Spicer 1999):

the conservation form of MHD equations for energy and momenta is based on the implicit $\boldsymbol{\nabla} \cdot \boldsymbol{B}=0$ condition, and all the topological aspects of magnetic field lines that are relevant to critical MHD phenomena, like reconnection, heavily rely on this condition.

On the other hand, this specific property has no easy representation in a numerical framework, like one-dimensional (1D) Godunov-type schemes, designed to handle compressive modes and shocks. This long-standing problem has been addressed by many authors, and several recipes have been proposed and experimented so far. Depending on the adopted methodology, these works can be broadly classified into three main categories:

1. Many MHD codes are constructed by simply extending to higher dimensions 1D Riemann solvers using a directional splitting technique, as in the Euler system (for second-order Godunov-type schemes: Zachary et al. 1994; Ryu, Jones, \& Frank 1995; Balsara 1998b; for fifth-order weighted essentially nonoscillatory (WENO) scheme: Jiang $\& \mathrm{Wu}$ 1999). In this approach the $\boldsymbol{\nabla} \cdot \boldsymbol{B}=0$ condition breaks down, of course, and some correction step has then to be applied. Following Brackbill \& Barnes (1980), a cleaning procedure is usually carried out by solving a Poisson equation, which is equivalent to adding a new (elliptic) equation to the original hyperbolic MHD system. As an empirical recipe, this method is by no means optimal and may lead to inconsistencies. In particular, the numerical derivatives appearing in Poisson equation have no clear relation with the upwind derivatives of the base MHD system and the boundary conditions become indeterminate for nontrivial boundary-value problems.

2. The Powell (1994) approach first pointed out the formal difficulty of applying 1D Riemann solvers to the multidimensional case, since the 1D MHD mode eigenspace, having seven components, is not of full rank of the two-dimensional (2D) case, in which an eight-component state vector is involved. Therefore, variations of magnetic field components appearing in the $\boldsymbol{\nabla} \cdot \boldsymbol{B}$ operator cannot be represented by Riemann solvers based on the 1D eigenspace. This undoubtedly correct premise led the author to propose a modification of the MHD equations by adding a " $\boldsymbol{\nabla} \cdot \boldsymbol{B}$ mode," propagating with the local flow speed. In this way the hyperbolic character of the MHD system is surely retained, at the price of suppressing the divergence-free property. This approach appears to be highly questionable, of course, since important physical properties of the MHD equations, and especially magnetic field topologies, are clearly lost.

3. In the present work we have taken as a main starting point all those references attempting to design upwind schemes in which a numerical divergence-free condition works as a build-in property (Evans \& Hawley 1988; DeVore 1991; Stone \& Norman 1992; Dai \& Woodward 1998; Ryu et al. 1998; Balsara \& Spicer 1999, among others). In all these works, the introduction of the magnetic vector potential or the equivalent conservative formulation of Stoke's theorem lead to represent magnetic field components at staggered collocation points, and a numerical $\boldsymbol{\nabla} \cdot \boldsymbol{B}=0$ relation follows as an algebraic identity. Moreover, when induction equations are properly formulated in terms of the staggered fields, conservation in time of the divergence-free property is also assured.

In the cited works, however, some main questions are still left open. These are essentially related to a persisting duality between staggered magnetic components evolving in the induction equations and the same components, now collocated at node points (or cell centers) as other fluid variables, entering the Riemann solver procedures. Several recipes based on interpolation have been suggested to relate cell centered and staggered fields. However, as extensively discussed in the Dai \& Woodward (1998) paper, the cell centered field components do not preserve, in general, the original divergence-free property, unphysical magnetic monopoles still arise, and their sizes seem to depend on the adopted interpolation schemes.

A related question concerns how upwind fluxes in the induction equations have to be formulated, since 1D Riemann solvers for density, momentum, and energy equations have no straightforward extension to them, when staggering is adopted. Again, many different empirical solutions have been proposed, which hardly can be compared and evaluated as long as only qualitative numerical tests are at disposal.

In the present paper we propose some general answers to these questions, by showing that consistency arguments are sufficient to envisage the main rules to adapt upwind schemes designed for Euler equations to the MHD case. Consistency requires that the specific operator structure of the MHD system and the related magnetic field properties have to be preserved by discretized equations and upwind procedures. In this way, different schemes and their highorder extensions can be designed, all assuring a numerical divergence-free condition as well as the related uniqueness and regularity of magnetic field lines. On the same ground, existing MHD codes and published numerical results can be evaluated on a more appropriate framework.

The plan of the paper is as follows. In $\S 2$ the general formulation to discretize Euler and MHD equations in conservation form is reviewed, with emphasis on the differences in space operator structures and on the related numerical representation. In $\S 3$, the kinematical properties of discontinuous, divergence-free magnetic fields are first analyzed and then used to construct appropriate upwind flux formulas (or approximate Riemann solvers) for the MHD equations. The proposed formulation is then also compared to recently published schemes. In $\S 4$ a code for $2 \mathrm{D}$ systems, based on third-order essentially nonoscillatory (ENO)-type reconstruction procedures and on the simple LaxFriedrichs flux upwinding, is presented. Section 5 is devoted to numerical test problems to add confidence and validation about the proposed approach, and concluding remarks are briefly given in $\S 6$.

\section{EULER VERSUS MHD SYSTEMS}

To underline differences between the Euler and MHD systems relevant to numerical discretization, we first briefly review some of the main points characterizing upwind schemes for the Euler equations. As a general framework, we consider here the flux vector splitting (FVS) formalism (van Leer 1982; Chen \& Lefloch 1995) and the high-order reconstruction techniques based on polynomials (Shu 1997). For ease of presentation we treat only the 2D case in Cartesian geometry, the three-dimensional (3D) case and the 
curvilinear geometries both being just straightforward extensions.

\subsection{Upwind Schemes for Euler Equations}

The equations of gasdynamics in two spatial dimensions constitute a system of $m=5$ conservation laws:

$$
\partial_{t} \boldsymbol{u}+\partial_{x}[\boldsymbol{f}(\boldsymbol{u})]+\partial_{y}[\boldsymbol{g}(\boldsymbol{u})]=0,
$$

where $\boldsymbol{u}=[\rho, \boldsymbol{q}, e]^{T}$ is the state vector of conservative variables and

$$
\begin{aligned}
& \boldsymbol{f}=\left[q_{x}, v_{x} q_{x}+p, v_{x} q_{y}, v_{x} q_{z}, v_{x}(e+p)\right]^{T}, \\
& \boldsymbol{g}=\left[q_{y}, v_{y} q_{x}, v_{y} q_{y}+p, v_{y} q_{z}, v_{y}(e+p)\right]^{T},
\end{aligned}
$$

are the corresponding flux vector functions. Here $\rho$ is the mass density, $\boldsymbol{q}=\rho \boldsymbol{v}$ the momentum associated with the flow velocity $v, e$ the total energy per unit volume, and $p=(\gamma-1)[e-\boldsymbol{q} \cdot \boldsymbol{v} / 2]$ the gas pressure for a $\gamma$-law equation of state.

A basic property of the Euler system is that each Jacobian matrix, $\boldsymbol{A}_{x}(\boldsymbol{u})=\partial_{\boldsymbol{u}} \boldsymbol{f}(\boldsymbol{u})$ and $\boldsymbol{A}_{y}(\boldsymbol{u})=\partial_{\boldsymbol{u}} \boldsymbol{g}(\boldsymbol{u})$, has a set of $m$ real eigenvalues $\left\{\lambda^{s}(\boldsymbol{u})\right\}(s=1,2, \ldots, m)$ and a corresponding complete sets of right $\left\{\boldsymbol{R}_{s}(\boldsymbol{u})\right\}$ and left $\left\{\boldsymbol{R}_{s}^{-1}(\boldsymbol{u})\right\}$ eigenvectors at every point $\boldsymbol{u}$ (hyperbolicity properties). Physically relevant solutions to system (1) are selected by imposing the admissibility condition

$$
\partial_{t}[\rho F(s)]+\nabla \cdot[\rho F(s) v] \leq 0,
$$

where $F(s)$ is any smooth function of the specific entropy $s(p, \rho)$ (Harten et al. 1998).

Numerical schemes for system (1) use the following consistency conditions as general guidelines (Tadmor 1988).

The conservation form, assuring that numerical solutions capture correctly weak solutions.

The entropy inequality, to be preserved by the discretized entropy functions. Upwind schemes are then designed to have (implicit) numerical viscosity compatible with relation (2).

In the semidiscrete formulation, appropriate for higher $r>2$ order schemes, space operators are approximated (for a fixed time $t$ ) on a $N_{x} \times N_{y}$ dimensional grid with node points $N_{j, k} \equiv\left(x_{j}, y_{k}\right)$, where $x_{j}=h_{x} j\left(j=0,1, \ldots, N_{x}-1\right)$ and $y_{k}=h_{y} k\left(k=0,1, \ldots, N_{y}-1\right)$; here $h_{x}$ and $h_{y}$ are the constant grid sizes along each direction. The point values formulation based on $\left\{\boldsymbol{u}_{j, k}\right\}$ data leads then to the conservative scheme

$$
\begin{aligned}
\frac{d \boldsymbol{u}_{j, k}(t)}{d t}=-\frac{1}{h_{x}}\left(\hat{\boldsymbol{f}}_{j+1 / 2, k}-\hat{\boldsymbol{f}}_{j-1 / 2, k}\right) & \\
& -\frac{1}{h_{y}}\left(\hat{g}_{j, k+1 / 2}-\hat{\boldsymbol{g}}_{j, k-1 / 2}\right),
\end{aligned}
$$

where $\hat{f}_{j+1 / 2, k}$ and $\hat{\boldsymbol{g}}_{j, k+1 / 2}$ denote the numerical flux-vector functions needed to approximate the corresponding flux derivatives to a given order $r$. A numerical approximation is then characterized essentially by the way $\hat{f}_{j+1 / 2, k}$ and $\hat{\boldsymbol{g}}_{j, k+1 / 2}$ are evaluated for a given set of $\left\{\boldsymbol{u}_{j, k}(t)\right\}$ data. Time integration can then be performed by appropriate RungeKutta or equivalent stable discretization schemes (Shu \& Osher 1988).

Modern higher order shock-capturing schemes generalize first-order Godunov scheme by following two main steps (Harten et al. 1987):
1. a reconstruction phase to recover variable values at grid points where flux derivatives have to be computed; and

2. an upwind phase, in which the Godunov method for a scalar conservation law in one dimension is extended to the $m>1$ components system in higher dimensions.

As far as item 1 is concerned, we review here some basic points relevant to the following sections and to the actual code structure, to be presented in $\S 4$.

Any one-dimensional piecewise smooth function $w(x)$, defined by cell-averaged data

$$
\bar{w}_{j}=\frac{1}{h_{x}} \int_{x_{j-1 / 2}}^{x_{j+1 / 2}} w(x) d x,
$$

can be approximated by uniform $(r-1)$ th order piecewise polynomials $P_{j}(x ; \bar{w})$, which must have the conservative property $\bar{P}_{j}=\bar{w}_{j}$. Likewise, a $w(x)$ function defined by gridpoint data $\left\{w_{j}=w\left(x_{j}\right)\right\}$ can be approximated by interpolation polynomials $P_{j}(x ; w)$ defined as $P_{j}\left(x_{j} ; w\right)=$ $w_{j}$. Here we denote with $R[x ; \bar{w}]$ (or $R[x ; w]$ ) the corresponding polynomials set $\left\{\boldsymbol{P}_{j}\right\}$ to reconstruct $w(x)$ at any $x$ point.

At points of discontinuity, $R[x ; \cdot]$ has to satisfy definite nonoscillatory constraints, for accuracy and stability purposes. Standard references are provided by linear polynomials based on min-mod limiters to preserve monotonicity of data (the monotone upwind scheme for conservation law [MUSCL] scheme: van Leer 1979; total variation diminishing [TVD] scheme: Harten 1983) or by higher $r>2$ order polynomials based on ENO procedures (Harten et al. 1987; Shu \& Osher 1989) having weaker (essentially nonoscillatory) monotonicity properties. Piecewise polynomials approximate the $w(x)$ function at any cell boundary point $x_{j+1 / 2}$ by a two-point left-right $\left(w^{(L)}, w^{(R)}\right)$ value, where

$$
\left(w^{(L)}\right)_{j+1 / 2}=P_{j}\left(x_{j+1 / 2}\right)=w\left(x_{j+1 / 2}^{-}\right)+O\left(h_{x}^{r}\right)
$$

and

$$
\left(w^{(R)}\right)_{j+1 / 2}=P_{j+1}\left(x_{j+1 / 2}\right)=w\left(x_{j+1 / 2}^{+}\right)+O\left(h_{x}^{r}\right),
$$

for smooth functions. For $w(x)$ having a discontinuous $k$ th derivative in the $x_{j}<x<x_{j+1}$ range, with $k<r$, the accuracy order becomes $O\left(h_{x}^{k+1}\right)$.

The reconstruction procedures can be extended to the $2 \mathrm{D}$ functions in equation (1) by assuming that the scalar components $u(x, y)$ (and hence $f[u(x, y)]$ and $g[u(x, y)])$ are piecewise smooth along each coordinate. In this way, calling $C_{j, k}$ the $2 \mathrm{D}$ cell centered at the node point $N_{j, k}$, the functions $u(x, y)$ can be reconstructed at the cell edges $\left(x_{j+1 / 2}, y_{k}\right)$ and $\left(x_{j}, y_{k+1 / 2}\right)$ by using, respectively, the 1D operators $R\left[x ; u_{k}\right]$ and $R\left[y ; u_{j}\right]$. The same functions may also be reconstructed at a cell corner $P_{j, k} \equiv$ $\left(x_{j+1 / 2}, y_{k+1 / 2}\right)$ by using the 2D compound operators $R\left[x_{j+1 / 2} ; R\left[y_{k+1 / 2} ; u\right]\right]$. Actually, space discretization in equation (1) involves only 1D reconstructions, one for each direction. In fact, $\hat{f}_{j+1 / 2, k}$ is defined at the point $x=x_{j+1 / 2}$, for fixed $y=y_{k}$, where the argument variables have the two-state $x$-wise reconstructed values $\left(u^{(L,)}, u^{(R,)}\right)_{k}$; likewise, $\hat{g}_{j, k+1 / 2}$ is defined at the point $y=y_{k+1 / 2}$, for fixed $x=x_{j}$, where the argument variables have the two-state $y$-wise reconstructed values $\left(u^{(, L)}, u^{(, R)}\right)_{j}$.

Let us now turn our attention to item 2. In the FVS formalism, to represent a flux variation, say, in the $x$ coordi- 
nate, the vector $f(u)$ is decomposed as

$$
f(u)=\frac{1}{2}\left(f^{(+)}+f^{(-)}\right), \quad f^{( \pm)}=f(u) \pm D_{x}(\tilde{u}) \cdot u,
$$

for states $\boldsymbol{u}$ around a given reference (constant) state $\tilde{\boldsymbol{u}}$. The flux vectors $\boldsymbol{f}^{( \pm)}$have Jacobian matrices $\boldsymbol{A}_{x}(\tilde{\boldsymbol{u}}) \pm \boldsymbol{D}_{x}(\tilde{\boldsymbol{u}})$ with only positive/negative eigenvalues. The matrix $\boldsymbol{D}_{x}$ is then required to be real (symmetrizable) and positive, $\boldsymbol{D}_{x}(\tilde{\boldsymbol{u}}) \geq$ $\left|\boldsymbol{A}_{x}(\tilde{\boldsymbol{u}})\right|$, where

$$
\left|\boldsymbol{A}_{x}(\tilde{\boldsymbol{u}})\right|=\sum_{s}\left(\boldsymbol{R}_{s}\left|\lambda_{s}\right| \boldsymbol{R}_{s}^{-1}\right)_{\tilde{\boldsymbol{u}}} .
$$

For given $\boldsymbol{u}_{1}=\boldsymbol{u}^{(L,)}, \boldsymbol{u}_{2}=\boldsymbol{u}^{(R,)}$ reconstructed values at the point $x_{j+1 / 2}$ and for fixed $k$ index, flux splitting allows one to define the numerical flux

$$
\begin{aligned}
f\left(\boldsymbol{u}_{1}, \boldsymbol{u}_{2}\right)_{j+1 / 2, k}=\frac{1}{2}\left[\boldsymbol{f}\left(\boldsymbol{u}_{1}\right)+\right. & \boldsymbol{f}\left(\boldsymbol{u}_{2}\right) \\
& \left.-\boldsymbol{D}_{x}(\tilde{\boldsymbol{u}}) \cdot\left(\boldsymbol{u}_{2}-\boldsymbol{u}_{1}\right)\right]_{j+1 / 2, k},
\end{aligned}
$$

which has upwind properties: namely, it is a two-point vector function nonincreasing in the first argument and nondecreasing in the second argument. The reference state $\tilde{\boldsymbol{u}}$ is given by the Roe average or by the simpler $\tilde{\boldsymbol{u}}=$ $\left(\boldsymbol{u}_{1}+\boldsymbol{u}_{2}\right) / 2$ arithmetic average, in a way to assure consistency $f(u, u)=f(u)$ and continuous dependence on data.

Standard references for the scheme in equation (4) are given by either the approximate Riemann solvers of Roetype or of Godunov-type , in which the corresponding matrix $D_{x}$ has the formal property

$$
\boldsymbol{D}_{x}(\tilde{\boldsymbol{u}})=\left|\boldsymbol{A}_{x}(\tilde{\boldsymbol{u}})\right|+O\left(\left|\boldsymbol{u}^{(L,)}-\boldsymbol{u}^{(R,)}\right|\right),
$$

entailing a minimum of numerical viscosity compatible with the entropy law. On the other hand, a maximum of numerical viscosity is achieved by the (global) Lax-Friedrichs (LF) flux, in which the $\boldsymbol{D}_{x}$ matrix reduces to the simple diagonal form

$$
\boldsymbol{D}_{x}(\tilde{\boldsymbol{u}})=\alpha \boldsymbol{I}, \quad \alpha=\max _{\tilde{\boldsymbol{u}}}\left(\max _{s}\left|\lambda_{s}(\tilde{\boldsymbol{u}})\right|\right),
$$

and in which Riemann characteristic informations are averaged out. Note that the flux formula equation (4) can be interpreted either as an approximate Riemann solver based on local linearization or as a discrete approximation of the associated viscosity model equation. In fact, a first-order approximation of the flux splitting is the discretized representation of the viscous flux

$$
\boldsymbol{f}_{v}(\boldsymbol{u})=\boldsymbol{f}(\boldsymbol{u})-h_{x} \boldsymbol{D}_{x} \cdot \partial_{x} \boldsymbol{u},
$$

which provides a link between the entropy condition and the numerical viscosity associated to the dissipation matrix $h_{x} \boldsymbol{D}_{x}$.

In $2 \mathrm{D}$ problems, the semidiscrete formulation of equation (3) and the independence of the Jacobian matrices $\left(\boldsymbol{A}_{x}, \boldsymbol{A}_{y}\right)$, allow one to represent the numerical fluxes $\hat{\boldsymbol{f}}_{j+1 / 2, k}$ (for fixed $y_{k}$ ) and $\hat{\boldsymbol{g}}_{j, k+1 / 2}$ (for fixed $x_{j}$ ) through independent upwind procedures, constructed with the matrices $\boldsymbol{D}_{x}$ and $\boldsymbol{D}_{y}$, respectively. In this way, the flux formula for $g$ is given by

$$
\begin{aligned}
g\left(u_{1}, u_{2}\right)_{j, k+1 / 2}=\frac{1}{2}[ & g\left(u_{1}\right)+g\left(u_{2}\right) \\
& \left.-D_{y}(\tilde{u}) \cdot\left(u_{2}-u_{1}\right)\right]_{j, k+1 / 2},
\end{aligned}
$$

where $\boldsymbol{u}_{1}=\boldsymbol{u}^{(, L)}$ and $\boldsymbol{u}_{2}=\boldsymbol{u}^{(, R)}$ are the reconstructed values at $y=y_{k+1 / 2}$ for fixed $x=x_{j}$. The corresponding viscosity form of the numerical flux $g$ is given by

$$
\boldsymbol{g}_{v}(\boldsymbol{u})=\boldsymbol{g}(\boldsymbol{u})-h_{y} D_{y} \cdot \partial_{y} \boldsymbol{u}
$$

The construction of upwind fluxes in equations (4) and (6), based on the $\boldsymbol{A}_{x}$ matrix eigenspace at the point $\left(x_{j+1 / 2}, y_{k}\right)$ and on the $\boldsymbol{A}_{y}$ matrix eigenspace at the point $\left(x_{j}, y_{k+1 / 2}\right)$, is usually referred to as a "directional splitting" setting. This procedure is consistent with the divergence form of space operators in equation (1) and implies that the space derivatives are obtained by summing the two flux differences computed both at the same time $t$. In the Strang-type formalism, which is widely adopted in the numerical astrophysics community, a splitting procedure is also applied to the time evolution operators, by constructing the updated solution of a $2 \mathrm{D}$ problem as a sequel of independent $1 \mathrm{D}$ problems, one for each direction, in turn. In the Euler system directional splitting or time splitting procedures give (formally) equivalent results, at least for second-order schemes. In the MHD case, however, this formal equivalence is definitely lost, as will be discussed in the following.

\subsection{The Two-dimensional MHD System}

The set of MHD conservation laws cannot be considered as a simple extension of the Euler system, with just a higher number of state variables. Actually, while the conservation form, the entropy law, and the general hyperbolic properties are maintained, some specific differences related to the structure of the space operators have to be considered.

In fact, the MHD system can be viewed as composed by two coupled subsystems, the first one containing space operators in the divergence form as in equation (1) evolving density, energy, and momenta, and the second one, specific to the magnetic field evolution, containing space operators in the curl form. In both subsystems the $\boldsymbol{\nabla} \cdot \boldsymbol{B}=0$ property of the vector magnetic field enters in a substantial way and has then to be considered as a new consistency condition for numerical discretization.

By specializing again to 2D systems, the MHD equations are given by

$$
\partial_{t} \boldsymbol{u}+\partial_{x}[\boldsymbol{f}(\boldsymbol{w})]+\partial_{y}[\boldsymbol{g}(\boldsymbol{w})]=0,
$$

for the six-component state vector $\boldsymbol{u}=\left[\rho, \boldsymbol{q}, e, B_{z}\right]^{T}$, coupled with the induction equations

$$
-\partial_{t} B_{x}+\partial_{y} \Omega(w)=0, \quad \partial_{t} B_{y}+\partial_{x} \Omega(w)=0,
$$

for the (poloidal) vector field $\boldsymbol{B}=\left[B_{x}, B_{y}\right]^{T}$. We denote the overall eight-component state vector as $\boldsymbol{w}=[\boldsymbol{u}, \boldsymbol{B}]^{T}$. The flux vectors in equation (8) are given by

$$
\begin{aligned}
\boldsymbol{f}(\boldsymbol{w}) & =\left[q_{x}, F_{x, x}, F_{x, y}, F_{x, z}, E_{x}, G_{x, z}\right]^{T}, \\
\boldsymbol{g}(\boldsymbol{w}) & =\left[q_{y}, F_{y, x}, F_{y, y}, F_{y, z}, E_{y}, G_{y, z}\right]^{T},
\end{aligned}
$$

where, for indices $i, j=x, y, z$,

$$
\begin{gathered}
F_{i, j}=v_{i} q_{j}-B_{i} B_{j}+\Pi \delta_{i, j}, \quad E_{i}=v_{i}(e+\Pi)-B_{i}\left(v_{j} B_{j}\right), \\
G_{i, j}=v_{i} B_{j}-v_{j} B_{i},
\end{gathered}
$$

in which the relations $F_{i, j} \equiv F_{j, i}$ and $G_{i, j} \equiv-G_{j, i}$ clearly hold. Here $\Pi=p+\left(B_{i} B_{i}\right) / 2$ and $p=(\gamma-1)\left[e-\left(q_{i} v_{i}\right) / 2\right.$ $\left.-\left(B_{i} B_{i}\right) / 2\right]$ are, respectively, the total and the gas pressures. The common flux function of equation (9) is defined as $\Omega=G_{x, y} \equiv-G_{y, x}=v_{x} B_{y}-v_{y} B_{x}$.

For given $\boldsymbol{B}$ field, the subsystem (8) is in Euler form, with independent Jacobian matrices of full $m=6$ rank, so the upwinding procedures based on directional splitting of the previous section can be extended. Differences arise, however, for the induction equations. In fact, subsystem (9) is generated by a unique flux function and has then only a 
one-dimensional eigenspace. This formal property is clearly related to the $\boldsymbol{\nabla} \cdot \boldsymbol{B}=0$ condition, as can be better evidenced by introducing a vector potential representation of the (poloidal) $\boldsymbol{B}$ components (here $A \equiv A_{z}$ )

$$
B_{x}=\partial_{y} A, \quad B_{y}=-\partial_{x} A .
$$

For given smooth $\left(B_{x}, B_{y}\right)$ fields, $A(x, y)$ always exists as a one-valued differentiable function. For discontinuous fields, equation (10) still holds in weak form, implying that $A(x, y)$ is at least (Lipschitz) continuous along each coordinate. On the other hand, for given $A(x, y, t)$, the system given by equation (9) is fully equivalent to the one-component evolution equation

$$
\partial_{t} A-\Omega(w)=0,
$$

coupled with equation (10).

In a formal setting, for given state variables $\boldsymbol{u}$, equation (11) is a Hamilton-Jacobi equation (Jin \& Xin 1998), and equation (9) represent the associated hyperbolic system. An important property of equation (11) is that $A(x, y, t)$ is continuous at all times and only discontinuities in its first derivatives may develop. Therefore, field lines defined by the isocontours $A(x, y)=$ const are allowed to have corners, but not jumps.

The overall MHD system can then be viewed as a coupled system of a Hamilton-Jacobi equation and of a set of conservation laws in the Euler form. One consequence is that the Jacobian matrix $\boldsymbol{A}_{x}$ corresponding to the $[f, \Omega]^{T}$ vector flux is only of $m=7$ rank and can represent characteristic modes of variables $\boldsymbol{w}_{x}=\left[\boldsymbol{u}(x,), B_{y}(x,)\right]^{T}$, while the independent matrix $\boldsymbol{A}_{y}$, corresponding to the $[g,-\Omega]^{T}$ vector flux, can represent variables $\boldsymbol{w}_{y}=\left[\boldsymbol{u}(, y), B_{x}(, y)\right]^{T}$. It is evident that the missing degrees of freedom $\left[B_{x}(x),, B_{y}(, y)\right]$ are not evolutionary and cannot have a characteristic-based representation.

A numerical scheme preserving these general properties must then be characterized by the following points.

1. A numerical $\boldsymbol{\nabla} \cdot \boldsymbol{B}=0$ condition and its conservation in time imply that the induction equation (9) have to be discretized using a unique flux function $\Omega(w)$ located at common points. This entails necessarily a staggered collocation of the magnetic field scalar components.

2. A divergence-free magnetic field is fully equivalent to its representation via a numerical vector potential and likewise the evolution equation (9), discretized as in item 1, can always be integrated via the scalar equation (11).

3. Relevant to the reconstruction and upwind steps is that the magnetic field components are at least continuous along the respective longitudinal coordinates, while discontinuities, to be related to the MHD characteristic modes, can occur only along the respective orthogonal coordinates (see below).

\section{3. $\boldsymbol{\nabla} \cdot \boldsymbol{B}=0$ PRESERVING UPWIND SCHEMES FOR MHD EQUATIONS}

In this section we concentrate on the correct collocation and reconstruction step for the numerical magnetic field data, and then on the upwind flux formulation for the induction equations, in order to preserve the peculiar features of the MHD system as outlined just above.

\subsection{The Reconstruction Step}

While for given data $\left\{u_{i, j}\right\}$ of $\boldsymbol{u}$ variables in equation (8) the reconstruction procedures follow the same lines as in the Euler system (3), for the field $\boldsymbol{B}$ it is necessary to take into account the $\boldsymbol{\nabla} \cdot \boldsymbol{B}=0$ condition as a new kinematical constraint. For general piecewise smooth fields this condition is expressed in integral form by

$$
\int_{\partial C} B_{n} d l=0
$$

for any cell $C$, where $B_{n}=\boldsymbol{B} \cdot \boldsymbol{n}$ and $\boldsymbol{n}$ is the unit vector normal to the boundary line $\partial C$. By first choosing a Cartesian cell with sides $\left(2 \epsilon, h_{y}\right)$, one finds the following continuity condition for the $y$-averaged $\bar{B}_{x}(x)$ component at any point $x$ :

$$
\bar{B}_{x}(x+\epsilon)-\bar{B}_{x}(x-\epsilon)=O(\epsilon) .
$$

The same argument leads to the continuity of the $x$-averaged $\bar{B}_{y}(y)$ field at any point $y$.

If equation (12) is then integrated over a computational cell $C_{j, k}$, one has

$$
\begin{aligned}
h_{y}\left[\bar{B}_{x}\left(x_{j+1 / 2}\right)-\right. & \left.\bar{B}_{x}\left(x_{j-1 / 2}\right)\right]_{k} \\
& +h_{x}\left[\bar{B}_{y}\left(y_{k+1 / 2}\right)-\bar{B}_{y}\left(y_{k-1 / 2}\right)\right]_{j}=0,
\end{aligned}
$$

where $\left(\bar{B}_{x}\right)_{k}$ is the $y$ average on the vertical cell side centered on $y_{k}$ and $\left(\bar{B}_{y}\right)_{j}$ the corresponding $x$ average over the horizontal cell side centered on $x_{j}$.

Continuity conditions and equation (13) are thus the main ingredients to construct at any point a divergence-free numerical field. In particular, the continuity condition $\bar{B}_{x}\left(x^{+}\right)=\bar{B}_{x}\left(x^{-}\right)$allows one to locate the $\left[\bar{B}_{x}(x)\right]_{k}$ field at cell boundary points $\left\{x_{j+1 / 2}\right\}$, where all the other variables $\left[\boldsymbol{w}_{x}\left(x_{j+1 / 2}\right)\right]_{k}$ are represented by reconstructed two-state (left-right) values. This property can be expressed in a formal way by setting $\left(\bar{B}_{x}^{(L,)}\right)_{j+1 / 2, k}=\left(\bar{B}_{x}^{(R,)}\right)_{j+1 / 2, k}$. Correspondingly, $\bar{B}_{y}(y)$ can be located at cell boundary points $\left\{y_{k+1 / 2}\right\}$ and point values can be interpreted as $\left(\bar{B}_{y}^{(, L)}\right)_{k+1 / 2}=\left(\bar{B}_{y}^{(, R)}\right)_{k+1 / 2}$.

The reconstruction step for the $\left(B_{x}, B_{y}\right)$ fields along the respective transverse coordinates leads to the point values

$$
\left[B_{x}(y)\right]_{j+1 / 2}=R\left[y ; \bar{B}_{x}\right], \quad\left[B_{y}(x)\right]_{k+1 / 2}=R\left[x ; \bar{B}_{y}\right],
$$

which have relevance for upwind computations. In fact, at the $y=y_{k+1 / 2}$ point, $B_{x}\left(y_{k+1 / 2}\right)=\left(B_{x}^{(, R)}, B_{x}^{(, L)}\right)$ is a two-state variable and can have only a transverse discontinuity line with a $\delta_{y} B_{x}=\left(B_{x}^{(, R)}-B_{x}^{(, L)}\right)$ jump. Similar arguments apply to the reconstructed values $B_{y}\left(x_{j+1 / 2}\right)=\left(B_{y}^{(R,)}, B_{y}^{(L,)}\right)$ at the $x=x_{j+1 / 2}$ boundary point, allowing only for a transverse discontinuity $\delta_{x} B_{y}=\left(B_{y}^{(R,)}-B_{y}^{(L,)}\right)$.

As already noticed by Evans \& Hawley (1988), a main property related to condition (13) is that the numerical data $\left\{\bar{B}_{x}, \bar{B}_{y}\right\}$ allow one to construct a unique (continuous) numerical vector potential $A\left(x_{j+1 / 2}, y_{k+1 / 2}\right)$, located at the cell corners $P_{j, k}$. It is evident that the reverse condition also holds true if one first introduces a numerical continuous function $A\left(x_{j+1 / 2}, y_{k+1 / 2}\right)$ and then defines the averaged magnetic field components through equation (10):

$$
\begin{aligned}
{\left[\bar{B}_{x}\left(x_{j+1 / 2}\right)\right]_{k} } & =\frac{1}{h_{y}}\left[\Delta_{y} A\left(x_{j+1 / 2}\right)\right]_{k}, \\
{\left[\bar{B}_{y}\left(y_{k+1 / 2}\right)\right]_{j} } & =-\frac{1}{h_{x}}\left[\Delta_{x} A\left(y_{k+1 / 2}\right)\right]_{j},
\end{aligned}
$$

where $\left(\Delta_{x}, \Delta_{y}\right)$ denote the usual (undivided) centered finite differences on the first and second coordinate indexes, respectively. In this way the divergence-free condition, 
equation (13), is identically satisfied by the commutativity of the $\Delta_{x}$ and $\Delta_{y}$ linear operators, whereas the continuity conditions follow from the continuity of $A(x, y)$. These arguments show, in particular, that the staggered collocation for $\left\{\bar{B}_{x}, \bar{B}_{y}\right\}$ data is by no means a numerical trick but arises in a consistent way from equation (13) or equation (14).

Equation (13) gives the cell average of the $\boldsymbol{\nabla} \cdot \boldsymbol{B}=0$ condition, and thus it is an exact law for second-order accurate schemes since $B_{x}=\bar{B}_{x}+O\left(h_{y}^{2}\right)$ at any point $\left(x_{j+1 / 2}, y_{k}\right)$ and $B_{y}=\bar{B}_{y}+O\left(h_{x}^{2}\right)$ at the corresponding staggered point. Now, if higher order approximation of point-valued $B_{x}$ and $B_{y}$ fields were recovered using independent reconstruction steps based on $\bar{B}_{x}$ and $\bar{B}_{y}$ data, a numerical $\boldsymbol{\nabla} \cdot \boldsymbol{B}$ of any size could arise, in general, since $1 \mathrm{D}$ reconstruction operators do not commute. To overcome this main difficulty, a different strategy has to be adopted, by first reconstructing accurate first derivatives based on the vector potential representation and having a numerical $\boldsymbol{\nabla} \cdot \boldsymbol{B}=0$ relation as a build-in property. As an illustration, in the following we consider third-order interpolations, but extensions to higher order can be easily pursued.

We first notice that cell averaged $\bar{w}_{j}$ and point values $w_{j}$ data of a given $w(x)$ function are related by $\bar{w}_{j}=w_{j}+$ $\gamma_{1} \mathscr{D}_{x}^{(2)}(w)_{j}+O\left(h^{3}\right)$, where $\gamma_{1}=1 / 24$ and $\mathscr{D}_{x}^{(2)}$ denotes a nonoscillatory numerical second derivative along the $x$ coordinate. To the same accuracy, the inverse relation $w_{j}=$ $\bar{w}_{j}-\gamma_{1} \mathscr{D}_{x}^{(2)}(\bar{w})_{j}$ approximates point values using averaged data. This algorithm, now applied to the values $w_{j+1 / 2}$ at cell interfaces, $\hat{w}_{j+1 / 2}=\left[w-\gamma_{1} \mathscr{D}_{x}^{(2)}(w)\right]_{j+1 / 2}$, gives the numerical $\hat{w}(x)$ primitive function whose two-point difference $\left(\Delta_{x} \hat{w}\right)_{j} / h_{x}$ constitutes a third-order approximation of the $\partial_{x} w(x)$ first derivative at $x_{j}$. Let then apply this reconstruction step to approximate the primitive $\hat{A}$ of the magnetic potential $A\left(x_{j+1 / 2}, y_{k+1 / 2}\right)$. In the 2D $(x, y)$ plane we have

$$
(\hat{A})_{j+1 / 2, k+1 / 2}=\left[A-\gamma_{1}\left(\mathscr{D}_{x}^{(2)}+\mathscr{D}_{y}^{(2)}\right) A\right]_{j+1 / 2, k+1 / 2},
$$

and the numerical magnetic field components are

$$
\begin{aligned}
\left(\hat{B}_{x}\right)_{j+1 / 2, k} & =\frac{1}{h_{y}}\left(\Delta_{y} \hat{A}\right)_{j+1 / 2, k}, \quad\left(\hat{B}_{y}\right)_{j, k+1 / 2} \\
& =-\frac{1}{h_{x}}\left(\Delta_{x} \hat{A}\right)_{j, k+1 / 2} .
\end{aligned}
$$

By definition, the difference $\left(\Delta_{x} \hat{B}_{x}\right) / h_{x}$ gives a third-order approximation of the $\partial_{x} B_{x}$ first derivative at the node $\left(x_{j}, y_{k}\right)$ point, and $\left(\Delta_{y} \hat{B}_{y}\right) / h_{y}$ gives the corresponding approximation of the $\partial_{y} B_{y}$ derivative with the same accuracy and at the same point. We notice that no left or right derivatives are defined along the longitudinal coordinates; thus these numerical approximations are unique. Moreover, one easily verifies that $\boldsymbol{\nabla} \cdot \boldsymbol{B}=0$, now in the point-valued form, is exactly fulfilled because of the commutativity of the $\Delta_{x} \Delta_{y}$ operator. The key point here is that to higher orders only the primitives $\left[\hat{B}_{x}(x),, \hat{B}_{y}(, y)\right]$ can be reconstructed directly using a common magnetic potential function $\hat{A}(x, y)$, but not the $\left(B_{x}, B_{y}\right)$ functions themselves (those entering the fluxes, where divergence-free fields are actually needed). To achieve this, one needs a further computational step, that is, to solve the (now implicit) relations

$$
\begin{aligned}
& \left(B_{x}-\gamma_{1} \mathscr{D}_{x}^{(2)} B_{x}\right)_{j+1 / 2, k}=\left(\hat{B}_{x}\right)_{j+1 / 2, k}, \\
& \left(B_{y}-\gamma_{1} \mathscr{D}_{y}^{(2)} B_{y}\right)_{j, k+1 / 2}=\left(\widehat{B}_{y}\right)_{j, k+1 / 2},
\end{aligned}
$$

where on the left-hand sides appear the primitives, and hence the derivatives, defined in terms of the (unknown) field point values, while on the right-hand sides are the source terms $\left(\hat{B}_{x}, \hat{B}_{y}\right)$, given by equation (15). The numerical fields $\left(B_{x}, B_{y}\right)$ defined by these equations and the corresponding (longitudinal) derivatives are third-order approximations but satisfy the divergence-free condition exactly. In practice one can solve equation (16) by some explicit iterative algorithm, since each operator $\left(1-\gamma_{1} \mathscr{D}^{(2)}\right)$ is clearly invertible, and a fourth-order accurate, at least, $\boldsymbol{\nabla} \cdot \boldsymbol{B}=O\left(h_{x}^{4}, h_{y}^{4}\right)$ condition can then be easily satisfied (see $\S$ 4).

This completes the main proof for the reconstruction step, needed to represent magnetic field point values in the momentum and energy equations, in which longitudinal derivatives and hence a $\boldsymbol{\nabla} \cdot \boldsymbol{B}=0$ condition has to be satisfied to avoid numerical monopoles. Moreover, for given $\left(B_{x}\right)_{j+1 / 2, k}$ and $\left(B_{y}\right)_{j, k+1 / 2}$ data it is possible to get interpolated values at other collocation points, where these field components act as independent variables and no divergence-free condition is then required.

\subsection{The Upwind Procedures}

Let us now consider the first set of MHD equation (8) discretized as in equation (3):

$$
\frac{d \boldsymbol{u}_{j, k}(t)}{d t}=-\frac{1}{h_{x}}\left(\hat{\boldsymbol{f}}_{j+1 / 2, k}-\hat{\boldsymbol{f}}_{j-1 / 2, k}\right)-\frac{1}{h_{y}}\left(\hat{\boldsymbol{g}}_{j, k+1 / 2}-\hat{\boldsymbol{g}}_{j, k-1 / 2}\right),
$$

where now the flux functions $[f(w), g(w)]$ depend on the eight-component vector $\boldsymbol{w}=[\boldsymbol{u}, \boldsymbol{B}]$. The upwind flux based on the $\boldsymbol{A}_{x}$ characteristic eigenspace has the form (consult eq. [4])

$$
\begin{aligned}
{\left[f\left(\boldsymbol{w}_{x}, B_{x}\right)\right]_{j+1 / 2, k} } & =\frac{1}{2}\left[\boldsymbol{f}\left(\boldsymbol{w}_{x}^{(R,)}, B_{x}\right)+\boldsymbol{f}\left(\boldsymbol{w}_{x}^{(L,)}, B_{x}\right)\right. \\
& \left.-\boldsymbol{D}_{x}^{(1-6)}(\tilde{\boldsymbol{w}}) \cdot\left(\boldsymbol{w}_{x}^{(R,)}-\boldsymbol{w}_{x}^{(L,)}\right)\right]_{j+1 / 2, k},
\end{aligned}
$$

where now upwind properties involve only the variables $\boldsymbol{w}_{x}=\left(\boldsymbol{u}, B_{y}\right)$. In the same way, the upwind flux based on the $\boldsymbol{A}_{y}$ characteristic eigenspace has the form (consult eq. [6])

$$
\begin{aligned}
{\left[\boldsymbol{g}\left(\boldsymbol{w}_{y}, B_{y}\right)\right]_{j, k+1 / 2} } & =\frac{1}{2}\left[\boldsymbol{g}\left(\boldsymbol{w}_{y}^{(, R)}, B_{y}\right)+\boldsymbol{g}\left(\boldsymbol{w}_{y}^{(, L)}, B_{y}\right)\right. \\
& \left.-\boldsymbol{D}_{y}^{(1-6)}(\tilde{\boldsymbol{w}}) \cdot\left(\boldsymbol{w}_{y}^{(, R)}-\boldsymbol{w}_{y}^{(, L)}\right)\right]_{j, k+1 / 2},
\end{aligned}
$$

where this time the upwind properties involve only the variables $\boldsymbol{w}_{y}=\left(\boldsymbol{u}, B_{x}\right)$.

To second-order approximation, the numerical fluxes needed to compute space derivatives in equation (17) are given by the flux values of equations (18) and (19), whose arguments $\boldsymbol{w}_{x}$ and $\boldsymbol{w}_{y}$ are second-order interpolated variables. In particular $B_{x}=\bar{B}_{x}$ in the $f\left(w_{x}, B_{x}\right)$ flux and $B_{y}=$ $\bar{B}_{y}$ in the $\boldsymbol{g}\left(\boldsymbol{w}_{y}, B_{y}\right)$ flux.

On the other hand, in classical second-order schemes for the Euler equations, the numerical flux $\hat{f}$ is expressed using cell-centered variables as

$$
\begin{aligned}
\hat{f}_{j+1 / 2, k}= & \frac{1}{2}\left[f\left(w_{j+1, k}\right)+f\left(w_{j, k}\right)\right] \\
& -\frac{1}{2}\left[D_{x}^{(1-6)}(\tilde{w}) \cdot\left(w_{x}^{(R,)}-w_{x}^{(L,)}\right)\right]_{j+1 / 2, k}
\end{aligned}
$$

and in a similar way for the $\boldsymbol{g}$ flux. However, this flux representation cannot be extended to the MHD case since cellcentered $\left(B_{x}, B_{y}\right)$ fields are not related by a divergence-free condition. The same remark applies to higher $r>2$ order 
schemes in which the numerical flux reconstruction is based on cell-centered values (Shu \& Osher 1989).

A second consequence of MHD structure in the evolution equation (17) is that the $u$ state vector has to be integrated in time by summing flux derivatives evaluated at the same time $t$, when the implicit $\boldsymbol{\nabla} \cdot \boldsymbol{B}=0$ condition holds. In those schemes in which time integration is performed by a Strangtype splitting procedure, flux derivatives and hence terms containing $\partial_{x} B_{x}$ and $\partial_{y} B_{y}$ are necessarily summed at different time steps and the required $\partial_{x} B_{x}+\partial_{y} B_{y}$ cancellation never occurs.

To summarize, higher order upwind schemes developed for Euler equations can be extended to the MHD subsystem (17), with the provisos that

1. flux derivatives have to be computed using flux values and hence $\boldsymbol{B}$ field data directly collocated at staggered (i.e., cell boundary centered) points and not at the cell centered points, and

2. the same derivatives along the two directions have to be computed at the same time, thus avoiding time-splitting techniques.

As already anticipated, the second set of MHD equations, given by the induction equation (9) for the magnetic poloidal field components, needs a particular treatment. In the proper, divergence-free preserving discretized form, these equations are given by

$$
\begin{aligned}
& \frac{d}{d t}\left[\bar{B}_{y}(t)\right]_{j, k+1 / 2}=-\frac{1}{h_{x}}\left[\Delta_{x} \Omega\left(w_{P}\right)\right], \\
& \frac{d}{d t}\left[\bar{B}_{x}(t)\right]_{j+1 / 2, k}=\frac{1}{h_{y}}\left[\Delta_{y} \Omega\left(w_{P}\right)\right],
\end{aligned}
$$

where now $\boldsymbol{w}=\left[v_{x}, v_{y}, B_{x}, B_{y}\right]^{T}$ denotes only the variables that are arguments of $\Omega$. In a fully equivalent form using the vector potential representation, one has

$$
\frac{d}{d t}[A(t)]_{P}=\Omega\left(w_{P}\right),
$$

to be coupled with equation (14). In both formulations a common flux function $\Omega(w)$, located at the cell corner point $P=\left(x_{j+1 / 2}, y_{k+1 / 2}\right)$, has to be evaluated.

In order to single out a consistent numerical flux function in equation (21), one has to take into account that $\Omega\left(\boldsymbol{w}_{P}\right)$ is now a four-state function

$$
\Omega^{(a, b)}=\Omega\left(w^{(a, b)}\right), \quad a, b=R, L,
$$

and upwind rules involve necessarily both $\boldsymbol{A}_{x}(\tilde{\boldsymbol{w}})$ and $\boldsymbol{A}_{y}(\tilde{\boldsymbol{w}})$ matrix eigenspaces evaluated at a common reference state $[\tilde{w}]_{P}$. To construct a proper 2D Riemann flux formula, we first consider the two limiting cases in which a propagating discontinuity may be described by a 1D Riemann flux formula.

1. For a discontinuity front perpendicular to the $x$ direction, where $w^{(, L)}=w^{(, R)}$, one has

$$
\begin{aligned}
{\left[\Omega_{x}(\boldsymbol{y})\right]_{j+1 / 2}=\frac{1}{2}\left[\Omega\left(\boldsymbol{w}^{(R,)}\right)+\right.} & \Omega\left(\boldsymbol{w}^{(L,)}\right) \\
& \left.-\boldsymbol{D}_{x}^{(7)}(\tilde{\boldsymbol{w}}) \cdot \delta_{x} \boldsymbol{w}_{x}\right](y)_{j+1 / 2},
\end{aligned}
$$

where $\boldsymbol{D}_{x}^{(7)}$ denotes the seventh row vector component of $\boldsymbol{D}_{x}$ matrix and $\delta_{x} w_{x}=\left(w_{x}^{(R,)}-w_{x}^{(L,)}\right)$. The flux in equation (22) is extended to the range $y_{k} \leq y \leq y_{k+1}$ where the variables $w^{(a)}(y), a=L, R$ are continuous. We remind the reader here that the upwind formula (22) can be derived from the flux splitting introduced in $\S 2.1$, in the form

$$
\Omega^{( \pm,)}=\Omega(w) \pm D_{x}^{(7)}(\tilde{w}) \cdot w_{x},
$$

which is equivalent to a local linearization of the $\Omega(w(x))$ flux around the $x=x_{j+1 / 2}$ point.

2. For a discontinuity front perpendicular to the $y$ direction, where now $\boldsymbol{w}^{(L,)}=\boldsymbol{w}^{(R,)}$, the approximate Riemann solver reduces to

$$
\begin{aligned}
{\left[\Omega_{y}(x)\right]_{k+1 / 2}=\frac{1}{2}\left[\Omega\left(\boldsymbol{w}^{(, R)}\right)\right.} & +\Omega\left(\boldsymbol{w}^{(, L)}\right) \\
& \left.+\boldsymbol{D}_{y}^{(7)}(\tilde{\boldsymbol{w}}) \cdot \delta_{y} \boldsymbol{w}_{y}\right](x)_{k+1 / 2},
\end{aligned}
$$

where $\boldsymbol{D}_{y}^{(7)}$ denotes the seventh row vector component of the $D_{y}$ matrix and $\delta_{y} \boldsymbol{w}_{y}=\left(\boldsymbol{w}_{y}^{(, R)}-\boldsymbol{w}_{y}^{(, L)}\right)$. The $x$ range involved is now $x_{j} \leq x \leq x_{j+1}$, where the variables $w^{(b)}(x)$, $b=L, R$ are continuous. Again, the upwind formula equation (24) may be derived from the splitting

$$
\Omega^{(, \pm)}=\Omega(w) \mp D_{y}^{(7)}(\tilde{w}) \cdot w_{y},
$$

which represents a local linearization around the $y=y_{k+1 / 2}$ point.

In the general case in which a discontinuity front crosses the computational cell centered on $P$, an approximate Riemann solver can be obtained by introducing a 2D flux splitting. For that purpose, we decompose each $\Omega^{( \pm,)}$component in equation (23) along the $y$ direction, with the requirement to have the same form of the symmetric decomposition of the $\Omega^{( \pm)}$components in equation (23) along the $x$ direction. This compound flux splitting, when interpreted as an approximate Riemann solver with local linearization [thus implying neglect of $O\left(\delta_{x} \boldsymbol{w}_{x} \delta_{y} \boldsymbol{w}_{y}\right)$ terms], results in the four-state flux formula

$$
\begin{aligned}
\Omega\left(\boldsymbol{w}_{P}\right)= & \frac{1}{4}\left[\Omega\left(\boldsymbol{w}^{(R, R)}\right)+\Omega\left(\boldsymbol{w}^{(R, L)}\right)+\Omega\left(\boldsymbol{w}^{(L, R)}\right)+\Omega\left(\boldsymbol{w}^{(L, L)}\right)\right]_{P} \\
& -\frac{1}{2}\left[\boldsymbol{D}_{x}^{(7)}\left(\tilde{\tilde{\boldsymbol{w}})} \cdot \delta_{x} \tilde{\boldsymbol{w}}_{x}-\boldsymbol{D}_{y}^{(7)}(\tilde{\tilde{\boldsymbol{w}}}) \cdot \delta_{y} \tilde{\boldsymbol{w}}_{y}\right]_{P}\right.
\end{aligned}
$$

where $(a, b=R, L)$ :

$$
\tilde{\boldsymbol{w}}_{x}^{(a,)}=\frac{1}{2}\left(\boldsymbol{w}_{x}^{(a, R)}+\boldsymbol{w}_{x}^{(a, L)}\right), \quad \tilde{\boldsymbol{w}}_{y}^{(, b)}=\frac{1}{2}\left(\boldsymbol{w}_{y}^{(R, b)}+\boldsymbol{w}_{y}^{(L, b)}\right),
$$

and $\quad \tilde{\tilde{w}}=\left(w^{(R, R)}+w^{(R, L)}+w^{(L, R)}+w^{(L, L)}\right) / 4$. The $\Omega(w)$ numerical flux given in equation (26) has now all the desired formal upwind properties both along the $x$ and $y$ directions and reduces correctly to the $1 \mathrm{D}$ limiting cases equations (22) and (24). The composition rule used here cannot be interpreted as a simple arithmetic average of independent 1D Riemann solvers. In fact, for a discontinuity front with arbitrary slope angle around $P$, the 1D flux formula, say equation (22), still applies and gives the upwind contribution along the $x$ characteristic modes. Near the $y=y_{k+1 / 2}$ point, $\Omega_{x}^{(, b)}(b=L, R)$ is now a two-state flux function and upwinding has to be completed by taking into account also the $-\boldsymbol{A}_{\boldsymbol{y}}$ characteristic modes along the orthogonal $y$ direction. By applying then 1D flux upwinding as in equation (24) to the flux $\Omega(w)=\Omega_{x}(w)$ and by discarding quadratic terms, one recovers equation (26). This composition procedure taken in reverse order, starting now from equation (24), yields an identical result under the essential assumption of linearization.

Finally, it is worth noticing that the viscous (resistive) model equation for the numerical flux function $\Omega$, consis- 
tent with equation (26), has the form

$$
\Omega_{v}(w)=\Omega(w)-\frac{1}{2}\left[h_{x} D_{x}(w) \cdot \partial_{x} w_{x}-h_{y} D_{y}(w) \cdot \partial_{y} w_{y}\right],
$$

showing how the dissipative term generalizes the classical $\eta \boldsymbol{J}=\eta \boldsymbol{\nabla} \times \boldsymbol{B}$ term in Ohm's law of resistive plasmas.

Having now completed the construction of the $\Omega(w)$ upwind flux for the induction equations, it is possible to design the overall numerical procedure to integrate the MHD system. We summarize here the main computational steps.

1. At each stage of the Runge-Kutta cycle, for given $(\boldsymbol{u}, A)(t)$ data at time $t$, the averaged $\left(\bar{B}_{x}, \bar{B}_{y}\right)$ staggered fields are evaluated first by equation (14).

2. Using then the $\left[u, \bar{B}_{x}, \bar{B}_{y}\right]^{T}$ data, all variables $\left[u, B_{x}, B_{y}\right]^{T}$ needed to compute the fluxes defined in equation (18) are reconstructed at each cell boundary point $\left(x_{j+1 / 2}, y_{k}\right)$, and conservative $x$-derivatives of the $f$ flux can then be evaluated.

3. The complementary procedure, now to reconstruct all variables $\left[u, B_{x}, B_{y}\right]^{T}$ for the fluxes defined in equation (19) at each $\left(x_{j}, y_{k+1 / 2}\right)$ point, gives the conservative $y$ derivatives of the $g$ flux.

4. A final reconstruction to the $\left(x_{j+1 / 2}, y_{k+1 / 2}\right)$ corner point is needed to compute the $\Omega(w)$ numerical flux in equation (26). This allows the integration in time the vector magnetic potential $A(t)$ by equation (21).

The explicit representation of a divergence-free magnetic field via a vector potential has, among others, the advantage of an easy extension to three-dimensional configurations. In fact, in this case one has to discretize the constitutive relations

$$
\boldsymbol{B}=\nabla \times \boldsymbol{A}, \quad \boldsymbol{B}=\left[B_{x}, B_{y}, B_{z}\right]^{T}
$$

in weak form to generalize equation (14). The $A_{i}, i=x, y, z$ components and the corresponding flux functions $\Omega_{i}=$ $(v \times B)_{i}$ are now located at the 3D cell edge points $P_{i}$, each centered along the corresponding $i$ th coordinate but staggered with respect to the remaining two directions. The evolution equation (eq. [21]) readily generalizes to

$$
\frac{d\left[A_{i}(t)\right]_{P_{i}}}{d t}=\Omega_{i}\left(w_{P_{i}}\right),
$$

and the consistency arguments introduced in 2D case can be applied to define the composition rules for each $\Omega_{i}=$ $\Omega_{i}(w)$ scalar function since only two upwind directions, in turn, are now involved (i.e. each $\Omega_{i}$ involves a 2D Riemann solver).

\subsection{Comments and Discussion}

Some remarks are due in order to underline differences and analogies with other proposed MHD schemes, in particular those presented by Dai \& Woodward (1998, hereafter DW), by Ryu et al. (1998, hereafter RY), and by Balsara \& Spicer (1999, hereafter BS), all claiming to have "divergence-free preserving properties." These works are based on second-order either Godunov or Roe-type schemes, and a staggered discretization for the induction equation of the form of our equation (20) is used.

At a second-order level the averaged variables $\left(\bar{B}_{x}\right)_{j+1 / 2, k}$ and $\left(\bar{B}_{y}\right)_{j, k+1 / 2}$ can be interpreted as point-valued variables at the same cell boundary points [which we label " $\left(b_{x}, b_{y}\right)$ " fields, to conform to the DW and RY notation]. In $\S 3$ we have shown that these staggered, divergence-free variables, when used in momentum and energy equations, avoid the effects of numerical monopoles. On the contrary, in all the cited works, while evolving in time the staggered $\left(b_{x}, b_{y}\right)$ magnetic field, interpolation and upwinding procedures are still based on cell-centered $\left(B_{x}, B_{y}\right)_{j, k}$ variables, and unwanted compressive $\boldsymbol{\nabla} \cdot \boldsymbol{B}$ terms are then necessarily set up. Moreover, this "duality" in the magnetic field representation is considered to be unavoidable in the Godunov-type Riemann solvers formalism. For that reason, in the DW and BS papers in particular, much attention has been devoted to compare the different results produced by schemes advancing in time only the $\left(b_{x}, b_{y}\right)$ staggered variables, where $\left(B_{x}, B_{y}\right)$ work as interpolated variables, and schemes in which the $\left(B_{x}, B_{y}\right)$ cell-centered components, also, are (independently) evolved (as for standard Godunov procedures for Euler equations). Conclusions are mainly drawn at a qualitative level, and only in the DW paper are some numerical results on the $\boldsymbol{\nabla} \cdot \boldsymbol{B}$ variable constructed with the $\left(B_{x}, B_{y}\right)$ data presented, showing the onset of significant, even of $O(1)$ size, residuals.

In the present approach, we have demonstrated by analytical arguments that the compressive components arise either in cases in which cell-centered fields are evolved in time or they are simply given by interpolation. In fact, at the leading second-order interpolation used in the cited papers,

$$
\begin{gathered}
\left(B_{x}\right)_{j, k}=\frac{1}{2}\left[\left(b_{x}\right)_{j+1 / 2, k}+\left(b_{x}\right)_{j-1 / 2, k}\right], \\
\left(B_{y}\right)_{j, k}=\frac{1}{2}\left[\left(b_{y}\right)_{j, k+1 / 2}+\left(b_{y}\right)_{j, k-1 / 2}\right],
\end{gathered}
$$

one gets $O\left(h^{2}\right)$ (for smooth fields) residual when the $\boldsymbol{\nabla} \cdot \boldsymbol{B}$ variable is evaluated by centered first derivatives. This compressive component associated to the $\left(B_{x}, B_{y}\right)$ fields cannot be considered to be small (of the same order of the truncation error), since it is easy to show that even for higher order $(r>2)$ interpolation the leading $\nabla \cdot \boldsymbol{B}=O\left(h^{2}\right)$ term never cancels out.

Our analysis shows that cell-centered fields are not really needed in upwind differentiation, even for Godunov-type schemes. In fact, the MHD system structure relies on two different kinds of magnetic field variables, depending on differentiation coordinates. The first set, given by the $\left[b_{x}(x),, b_{y}(, y)\right]$ variables, which are continuous in the indicated coordinates, satisfies the divergence-free condition and does not have a characteristic representation. The second one, given by $\left[B_{x}(, y), B_{y}(x),\right]$, enters the characteristic space and can then have (transverse) discontinuities. The former quantities are advanced in time as staggered field data, while the latter can be reconstructed by interpolation either at a cell boundary or at a cell corner point.

As a last point, we comment here on the way the flux for the induction equations is derived. In the DW and BS schemes, the $\Omega$ fluxes (or electric fields components) are constructed by simple arithmetic averages in space and for each 1D upwind flux. This approach, which seems reasonable for second-order accuracy, is not consistent since it does not reduce to the original 1D fluxes when the discontinuity front propagates along one of the coordinate axes. This drawback led BS to introduce a rather empirical switch to select the dominant direction of front propagation. On the other hand, RY derives a formally correct flux, of the same form as our equation (26), by splitting the flux $\Omega=v_{x} B_{y}-v_{y} B_{x}$ into two independent components $\left(v_{x} B_{y}\right.$ and $v_{y} B_{x}$ ), and 1D independent upwindings along the $x$ and 
$y$ direction, respectively, have then been applied. However, this computational trick has no physical support, since the two characteristics spaces, spanned by the $\boldsymbol{A}_{x}$ and $\boldsymbol{A}_{y}$, in the original MHD equations, are both based on the complete $\Omega$ flux.

\section{IMPLEMENTATION OF A THIRD-ORDER LF-CENO SCHEME}

We consider the 2D MHD system in Cartesian $(x, y)$ coordinates, in the conservation form given by equation (17), to integrate the density, momenta, and energy variables, and by equation (21) to integrate the vector potential. The updated, line-averaged, magnetic fields $\left(\bar{B}_{x}, \bar{B}_{y}\right)$ are defined, at each time step, by the geometrical relations of equation (14).

We specify the general procedure outlined in the previous $\S 3$ by choosing high-order reconstruction algorithms based on convex-ENO (CENO) method, a local Lax-Friedrichs (LLF) flux splitting for upwinding, and a time integration step using a third-order TVD Runge-Kutta scheme.

All the indicated numerical ingredients are well documented and tested in problems described by the Euler system of gasdynamics (for time integration: Shu \& Osher 1988; for general ENO reconstruction: Shu 1997; for the CENO method: Liu \& Osher 1998). It is then sufficient to detail here the specific procedures allowing to extend CENO schemes to the MHD system and having relevance to the divergence-free properties of the magnetic fields.

1. Among high-order reconstruction algorithms, the recently proposed CENO method has the main computational advantage of avoiding the time-consuming characteristic decomposition of state variables, which is usually adopted in upwind schemes.To achieve this property, one consider first a TVD (monotone) second-order accurate interpolant for the scalar variable $w(x)$ with data $\left\{w_{j}\right\}$, where $w$ denotes any component of the state vector $w$. In the $x_{j-1 / 2} \leq x \leq x_{j+1 / 2}$ range, three-point linear polynomials have the form

$$
L_{j}^{(k)}(x)=w_{j}+\frac{1}{h_{x}}(\Delta w)_{j+k}\left(x-x_{j}\right), \quad k=0,1,
$$

where $(\Delta w)_{i}=w_{i+1}-w_{i}$. In classical TVD schemes a unique interpolant $\tilde{L}_{j}(x)=w_{j}+\left(1 / h_{x}\right)\left(\mathscr{D}^{(1)} w\right)_{j}\left(x-x_{j}\right)$ with slope $\left(\mathscr{D}^{(1)} w\right)_{j}$ is chosen using min-mod (" $\mathrm{mm}$ ") limiters to assure nonoscillatory properties

$$
\left(\mathscr{D}^{(1)} w\right)_{j}=\mathrm{mm}\left[(\Delta w)_{j-1},(\Delta w)_{j}\right] .
$$

The $\mathrm{mm}(a, b)$ function is defined, as usual, by

$$
\operatorname{mm}(a, b)=\operatorname{sign}(a) \min (|a|,|b|) \quad \text { for } a b>0
$$

and $\mathrm{mm}(a, b)=0$ otherwise.Using the selected $\tilde{L}_{j}(w)$ polynomial, the interpolated values at the cell boundaries are then given by $\left(w^{(L)}\right)_{j+1 / 2}=\tilde{L}_{j}\left(x_{j+1 / 2}\right), \quad\left(w^{(R)}\right)_{j+1 / 2}=$ $\tilde{L}_{j+1}\left(x_{j+1 / 2}\right)$.At any point of discontinuity as well at any smooth extrema of $w(x)$ where the first differences change sign, the TVD polynomial reduces to the constant state $L_{j}(x)=w_{j}$. Clipping to first-order accuracy at a function jump is unavoidable in any polynomial-based reconstruction, while higher $(r \geq 2)$ accuracy in smooth ranges can always be achieved by enforced TVD or ENO procedures. This improvement, however, usually requires a preliminary decomposition of the $\Delta w$ differences into characteristic modes, locally at each grid point. In the CENO method a higher order interpolation is maintained only at smooth regions while first-order polynomials are used at the function jumps, with neither case requiring a characteristic decomposition.For third-order reconstruction, in particular, one has at one's disposal three quadratic interpolants in the $x_{j-1 / 2} \leq x \leq x_{j+1 / 2}$ range

$$
\begin{aligned}
Q_{j}^{(k)}(x)= & w_{i}+\frac{1}{2}\left[(\Delta w)_{i}+(\Delta w)_{i-1}\right] \\
& \times \frac{\left(x-x_{i}\right)}{h_{x}}+\frac{1}{2}\left(\Delta^{(2)} w\right)_{i} \frac{\left(x-x_{i}\right)^{2}}{h_{x}^{2}},
\end{aligned}
$$

where $\left(\Delta^{(2)}\right)_{i}=\left(\Delta_{i}-\Delta_{i-1}\right)$ and centering refers to the index $i=j+k$ for $k=-1,0,1$. The CENO selection procedure allows to construct the unique nonoscillatory interpolant

$$
\tilde{Q}_{j}(x)=w_{j}+\frac{1}{2}\left(\mathscr{D}^{(1)} w\right)_{j} \frac{\left(x-x_{j}\right)}{h_{x}}+\frac{1}{2}\left(\mathscr{D}^{(2)} w\right)_{j} \frac{\left(x-x_{j}\right)^{2}}{h_{x}^{2}},
$$

which is closest to the TVD lower order interpolant $\tilde{L}_{j}(w)$. This is obtained by computing the three differences

$$
d_{j}^{(k)}=Q_{j}^{(k)}-\tilde{L}_{j}, \quad k=-1,0,1
$$

at the $x=x_{j-1 / 2}$ or $x=x_{j+1 / 2}$ boundary point. In a smooth range all these distance indicators have the same sign, and one can then select

$$
\tilde{Q}_{j}=Q_{j}^{\left(k_{0}\right)}, \quad\left|d^{\left(k_{0}\right)}\right|=\min _{k}\left|d^{(k)}\right| .
$$

Only at a discontinuity point at least one indicator changes sign and in this case one takes $\widetilde{Q}_{j}=\widetilde{L}_{j}=w_{j}$ since $\mathscr{D}^{(1)}=\mathscr{D}^{(2)}=0$, clipping to a first-order interpolation.

2. We apply then this reconstruction procedure, first to each $u_{j, k}$ scalar component of the $\boldsymbol{u}$ state vector, to recover the point values

$$
\begin{aligned}
\left(u^{(L)}\right)_{j+1 / 2, k} & =\widetilde{Q}_{j}(u)\left(x_{j+1 / 2}, y_{k}\right), \\
\left(u^{(R)}\right)_{j+1 / 2} & =\widetilde{Q}_{j+1}(u)\left(x_{j+1 / 2}, y_{k}\right),
\end{aligned}
$$

needed to compute the $f\left(w_{x}, B_{x}\right)$ flux, and in a similar way to recover the $u_{j, k+1 / 2}$ values needed to compute the $\boldsymbol{g}\left(\boldsymbol{w}_{y}, B_{y}\right)$ flux.

3. The definition of $\left(B_{x}, B_{y}\right)$ point values introduced in $\S 3$ requires the specification of the nonoscillatory $\mathscr{D}^{(2)}$ second derivative. In the CENO framework one simply takes

$$
\begin{aligned}
& \mathscr{D}_{x}^{(2)}=\mathrm{mm}\left(\Delta_{j-1}^{(2)}, \Delta_{j}^{(2)}, \Delta_{j+1}^{(2)}\right), \\
& \mathscr{D}_{y}^{(2)}=\mathrm{mm}\left(\Delta_{k-1}^{(2)}, \Delta_{k}^{(2)}, \Delta_{k+1}^{(2)}\right) .
\end{aligned}
$$

We notice that this procedure returns the smoothest among the indicated three second numerical derivatives if the stencil of the involved first differences $\left(\Delta_{j-2}, \ldots, \Delta_{j+1}\right)$ is monotone, while $\mathscr{D}^{(2)}=0$ if the first derivative has a jump or a smooth extremum.For given $\left(\hat{B}_{x}\right)_{j+1 / 2, k}$, the reconstruction of the related divergence-free $\left(B_{x}\right)_{j+1 / 2, k}$ point values is given by the implicit definition in equation (16)

$$
\left(B_{x}-\gamma_{1} \mathscr{D}_{x}^{(2)} B_{x}\right)_{j+1 / 2, k}=\left(\hat{B}_{x}\right)_{j+1 / 2, k}, \quad \gamma_{1}=1 / 24,
$$

which we solve by using an explicit iteration procedure. By setting $B_{x}^{(0)}=\hat{B}_{x}$, at each $\left(x_{j+1 / 2}, y_{k}\right)$ point, the sequence

$$
B_{x}^{(n)}=\hat{B}_{x}+\gamma_{1} \mathscr{D}_{x}^{(2)}\left(B_{x}^{(n-1)}\right), \quad n=1,2, \ldots,
$$


is clearly rapidly convergent since $\mathscr{D}_{x}^{(2)}$ is at most an $O\left(h_{x}\right)$ quantity. In a similar way, we compute $\left(B_{y}\right)_{j, k+1 / 2}$ by iterating

$$
B_{y}^{(n)}=\hat{B}_{y}+\gamma_{1} \mathscr{D}_{y}^{(2)}\left(B_{y}^{(n-1)}\right), \quad B_{y}^{(0)}=\hat{B}_{y} .
$$

In practical computations, for all the test problems presented in the next section, we found that $n=5$ is sufficient to assure $\boldsymbol{\nabla} \cdot \boldsymbol{B}=0$ to within machine accuracy both in the maximum and in the $L_{1}$ norm. The computed $\left(B_{x}\right)_{j+1 / 2, k}$ point values computed in equation (27) enter now the $f\left(w_{x}, B_{x}\right)$ flux as they stand, while the $\left(B_{y}\right)_{j+1 / 2, k}$ component of the $\boldsymbol{w}_{x}$ state vector needs further interpolation. Using then $\left(B_{y}\right)_{j, k+1 / 2}$ derived by equation (28), the cell-centered $\left(B_{y}\right)_{j, k}$ field is first reconstructed by taking

$$
\begin{aligned}
\left(B_{y}\right)_{j, k} & =\frac{1}{2}\left[\left(\widetilde{B}_{y}\right)_{j, k-1 / 2}+\left(\widetilde{B}_{y}\right)_{j, k+1 / 2}\right], \\
\left(\widetilde{B}_{y}\right)_{j, k+1 / 2} & =\left(B_{y}\right)_{j, k+1 / 2}-\frac{1}{8}\left(\mathscr{D}_{y}^{(2)} B_{y}\right)_{j, k+1 / 2},
\end{aligned}
$$

to be finally interpolated at the $\left(x_{j+1 / 2}, y_{k}\right)$ cell boundary point like the other $u_{j, k}$ components of the $\boldsymbol{w}_{x}$ state vector. It is worth noticing that the cell-centered $\left(B_{y}\right)_{j, k}$ values do not have divergence-free properties since the $f\left(w_{x}, B_{x}\right)$ differentiation involves only the $x$ coordinate.By symmetric arguments, the $\left(B_{y}\right)_{j, k+1 / 2}$ field enters the $g\left(w_{y}, B_{y}\right)$ flux as it stands, while $\left(B_{x}\right)_{j+1 / 2, k}$ now has to be interpolated using the cell-centered values

$$
\begin{aligned}
\left(B_{x}\right)_{j, k} & =\frac{1}{2}\left[\left(\widetilde{B}_{x}\right)+\left(\widetilde{B}_{y}\right)_{j+1 / 2, k}\right], \\
\left(\widetilde{B}_{x}\right)_{j+1 / 2, k} & =\left(B_{x}\right)_{j+1 / 2, k}-\frac{1}{8}\left(\mathscr{D}_{x}^{(2)} B_{x}\right)_{j+1 / 2, k} .
\end{aligned}
$$

4. The interpolated $\left(\boldsymbol{w}_{x}\right)_{j+1 / 2, k}$ or $\left(\boldsymbol{w}_{y}\right)_{j, k+1 / 2}$ are represented as two-point left-right values along the relevant $x$ or $y$ coordinate, and an approximate Riemann solver has then to be specified to compute upwind fluxes. We have chosen the simple LLF flux composition, defined by

$$
\boldsymbol{f}\left(\boldsymbol{w}_{x}, B_{x}\right)=\frac{1}{2}\left[\boldsymbol{f}\left(\boldsymbol{w}_{x}^{L,}, B_{x}\right)+\boldsymbol{f}\left(\boldsymbol{w}_{x}^{R,}, B_{x}\right)\right]-\frac{1}{2} \alpha_{x}\left(\tilde{\boldsymbol{w}}_{x}\right)\left(\boldsymbol{u}^{R,}-\boldsymbol{u}^{L,}\right)
$$

and

$$
g\left(w_{y}, B_{y}\right)=\frac{1}{2}\left[g\left(w_{y}^{L}, B_{y}\right)+g\left(w_{y}^{, R}, B_{y}\right)\right]-\frac{1}{2} \alpha_{y}\left(\tilde{w}_{y}\right)\left(u^{R}-u^{L}\right) .
$$

The scalar variable $\alpha_{x}\left(\tilde{\boldsymbol{w}}_{x}\right)$ is given at each $\left(x_{j+1 / 2}, y_{k}\right)$ point by the largest of the $\boldsymbol{A}_{x}$ matrix eigenvalues $\lambda_{s}\left(\tilde{\boldsymbol{w}}_{x}\right)$, and $\tilde{\boldsymbol{w}}_{x}$ is the arithmetic average of the $\boldsymbol{w}_{x}$ left-right states. In practice $\alpha_{x}=\left|v_{x}\right|+c_{f_{x}}$, where $c_{f_{x}}$ is the fast wave speed along the $x$ direction. Correspondingly, $\alpha_{y}\left(\tilde{\boldsymbol{w}}_{y}\right)=\left|v_{y}\right|+c_{f_{y}}$ gives the largest eigenvalue of the $\boldsymbol{A}_{y}$ matrix based on the arithmetic average of the left-right states of $\boldsymbol{w}_{y}$ at the $\left(x_{j}, y_{k+1 / 2}\right)$ point.

5. Differences of flux values given in equations (29) and (30) provide only second-order accurate derivatives, even if the reconstructed flux arguments share a higher accuracy order. To keep third-order in derivative approximations, we construct the primitives

$$
\begin{aligned}
\hat{\boldsymbol{f}}_{j+1 / 2, k} & =\left(\boldsymbol{f}-\gamma_{1} \mathscr{D}_{x}^{(2)} \boldsymbol{f}\right)_{j+1 / 2, k}, \quad \hat{\boldsymbol{g}}_{j, k+1 / 2} \\
& =\left(\boldsymbol{g}-\gamma_{1} \mathscr{D}_{y}^{(2)} \boldsymbol{g}\right)_{j, k+1 / 2},
\end{aligned}
$$

thus completing the integration scheme of equation (17) for the six-component state vector $\boldsymbol{u}_{j, k}$.

6 . The $\Omega(w)$ flux variable needs a proper upwinding procedure, as shown in equation (26). In an LLF scheme one
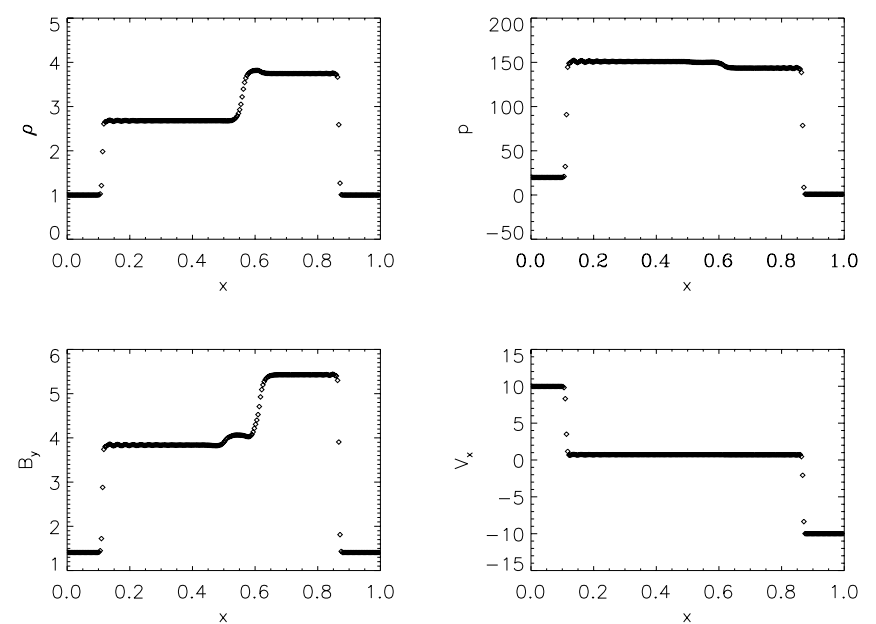

FIG. 1.- Indicated variables at time $t=0.08$ for the 1D Riemann problem RJ1, using $N_{x}=400$ grid points.

has

$$
\begin{gathered}
\Omega\left(w_{P}\right)=\frac{1}{4}\left[\Omega\left(w^{(R, R)}\right)+\Omega\left(w^{(R, L)}\right)+\Omega\left(w^{(L, R)}\right)+\Omega\left(w^{(L, L)}\right)\right]_{P} \\
-\frac{1}{2}\left[\alpha_{x}(\tilde{\tilde{w}}) \delta_{x} B_{y}-\alpha_{y}(\tilde{\tilde{w}}) \delta_{y} B_{x}\right]_{P},
\end{gathered}
$$

where all the arguments $\left(v_{x}, v_{y}, B_{x}, B_{y}\right)$ are first interpolated at a common $P=\left(x_{j+1 / 2}, y_{k+1 / 2}\right)$ point.

7. Finally, for time integration, a three-step Runge-Kutta algorithm provides the overall third-order accuracy of the LF-CENO scheme.

\section{NUMERICAL RESULTS}

The proposed numerical problems are mainly concerned with the divergence-free property, which on numerical side entails two main aspects:

1. the existence of a vector potential $A\left(x_{j+1 / 2}, y_{k+1 / 2}, t\right)$ as a continuous function at all times, assuring regular field lines topology (only corners are allowed), and

2. a vanishing $D_{B} \equiv(\nabla \cdot \boldsymbol{B})_{j, k}$, where field derivatives are computed using the same field components and the same difference algorithms as in dynamical flux calculations.

Since this form of validation has no counterpart in other proposed numerical works, comparisons with published
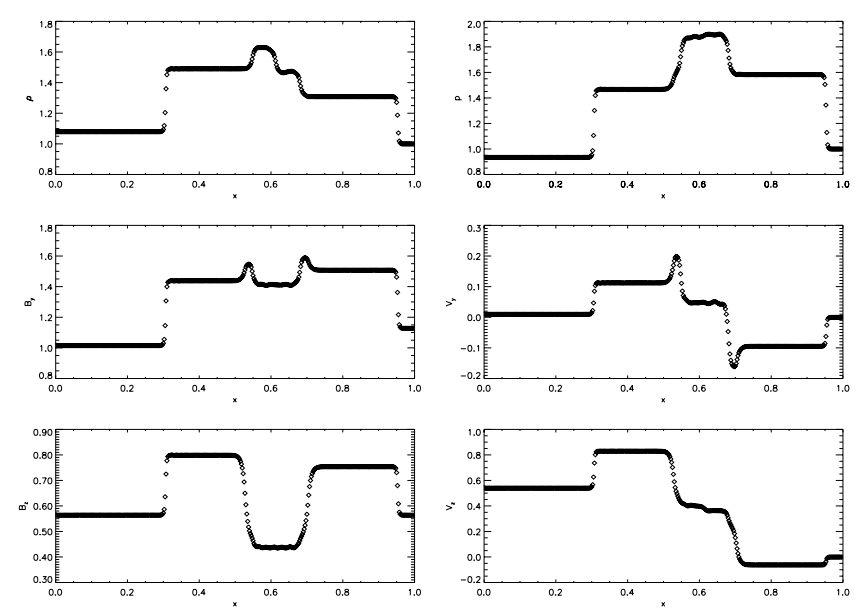

FIG. 2.-Indicated variables at time $t=0.2$ for the 1D Riemann problem RJ2, using $N_{x}=400$ grid points. 

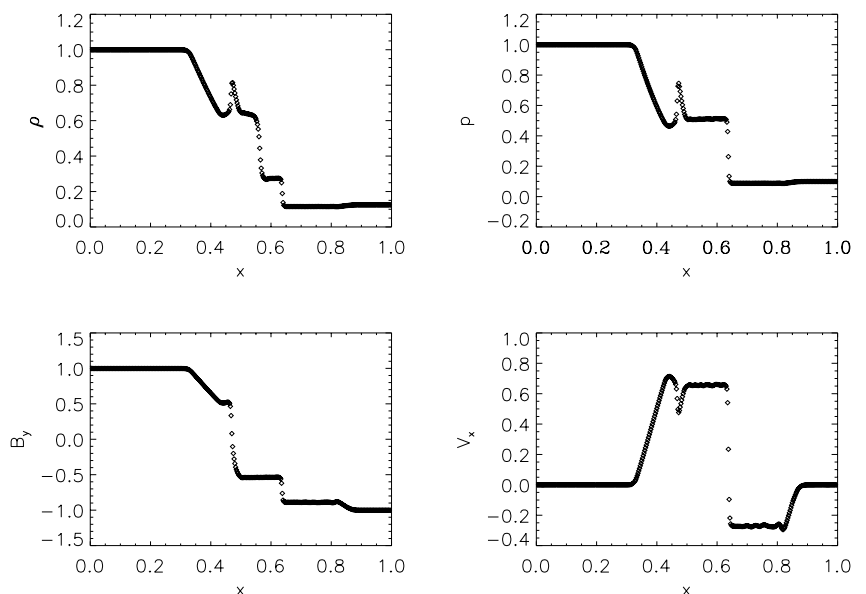

FIG. 3.-Indicated variables at time $t=0.1$ for the 1D Riemann problem RJ3, using $N_{x}=400$ grid points.

data will cover necessarily rather qualitative aspects. Beside the divergence-free condition, the numerical results give also indications on the resolution properties, as well as on the stability and reliability of the MHD code described in $\S 4$.

Finally, we remark that in our code the $A(x, y)$ vector potential refers only to the nonuniform $\left(B_{x}, B_{y}\right)$ fields, since constant initial components are trivially preserved in time. Therefore, for problems having constant components $\left(B_{0 x}, B_{0 y}\right)$, the evolved $A(t)$ field is now defined by

$$
B_{x}=B_{0 x}+\partial_{y} A, \quad B_{y}=B_{0 y}-\partial_{x} A,
$$

replacing the original equation (10) of $\S 2$.

\subsection{Shock-Tube Tests}

We first consider 1D Riemann problems (using a full 2D grid) to check for resolution properties of the proposed scheme. To that purpose, it is necessary to take into account that high-order schemes are not well suited for shock-tube problems in which lower order characteristic-based schemes are optimal, instead.

We consider three problems documented by Ryu \& Jones (1995) in their Figures 1a, 2a, and 5a, which we here label
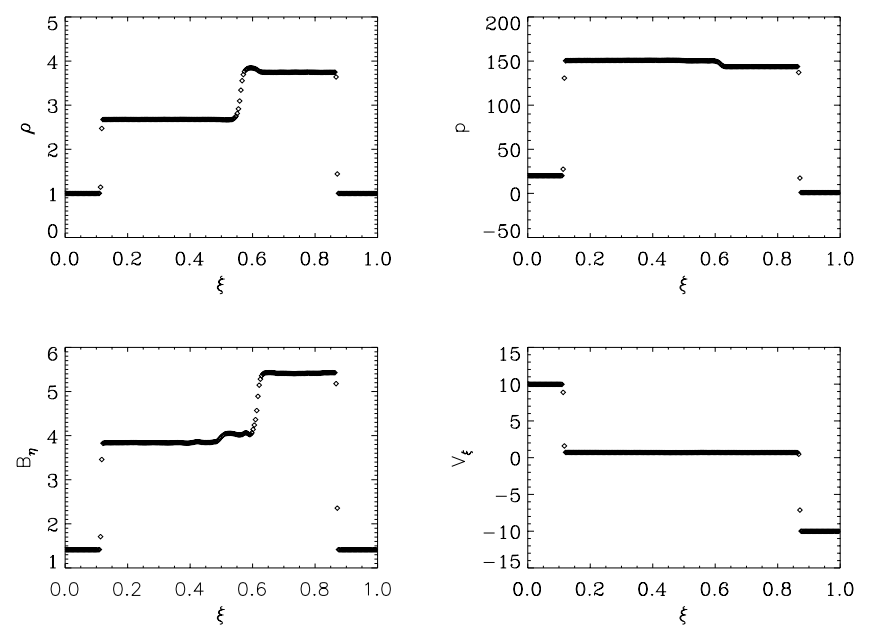

FIG. 4.- Same shock-tube problem as in Fig. 1, now along the main diagonal $\xi$ of a 2 D square computational box with $256 \times 256$ grid points.

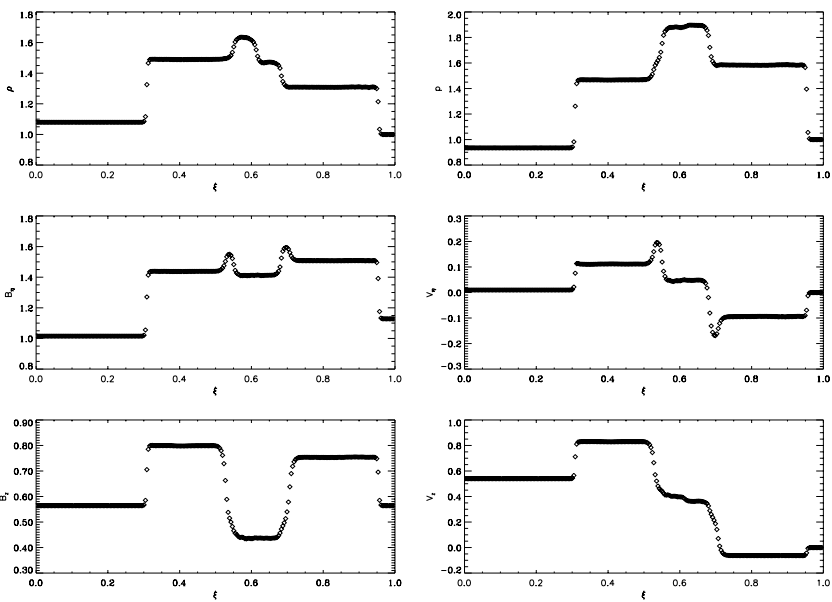

Fig. 5.-Same shock-tube problem as in Fig. 2, now along the main diagonal $\xi$ of a $2 \mathrm{D}$ square computational box with $256 \times 256$ grid points.

"RJ1," "RJ2," and "RJ3," respectively. In all the indicated cases a uniform grid with $N_{x}=400$ grid points, a grid size $L_{x}=1$, an adiabatic index $\gamma=5 / 3$, and a CourantFriedrichs-Levy (CFL) number $c=0.8$, are used. In all numerical tests presented here, the parameters $\gamma$ and $c$ will always retain the same values.

In RJ1, the initial conditions for the state vector $\boldsymbol{w}(x)=\left[\rho, v_{x}, v_{y}, v_{z}, B_{y}, B_{z}, p\right]^{T}$ are defined by

$$
\begin{aligned}
\boldsymbol{w}^{L} & =\left[1,10,0,0,5 B_{0}, 0,20\right]^{T}, \\
\boldsymbol{w}^{R} & =\left[1,-10,0,0,5 B_{0}, 0,1\right]^{T},
\end{aligned}
$$

and by a constant $B_{0 x}=5 B_{0}$. Here left states refer to $x<0.5$ and right states to $x>0.5$. The unit magnetic field is $B_{0}=(4 \pi)^{-1 / 2}$. In Figure 1 the evolved variables $(\rho, p$, $B_{y}, v_{x}$ ) are shown at time $t=0.08$, as in Ryu \& Jones (1995).

In the $\mathrm{RJ} 2$ test initial conditions are defined by

$$
\begin{aligned}
\boldsymbol{w}^{L} & =\left[1.08,1.2,0.01,0.5,3.6 B_{0}, 2 B_{0}, 0.95\right]^{T}, \\
\boldsymbol{w}^{R} & =\left[1,0,0,0,4 B_{0}, 2 B_{0}, 1\right]^{T},
\end{aligned}
$$

and the constant magnetic field is now $B_{0 x}=2 B_{0}$. The evolved variables $\left(\rho, p, B_{y}, v_{y}, B_{z}, v_{z}\right)$ at time $t=0.2$ are shown in Figure 2.

Finally, the RJ3 problem, with initial data

$$
\begin{aligned}
& \boldsymbol{w}^{L}=[1,0,0,0,1,0,1]^{T}, \\
& \boldsymbol{w}^{R}=[0.125,0,0,0,-1,0,0.1]^{T},
\end{aligned}
$$

and $B_{0 x}=0.75$, is illustrated in the Figure 3, for $t=0.1$. This is already considered a classical test, related to the presence of a compound wave (Brio \& Wu 1988).

As can be seen, the plotted results reproduce well all the main expected features and compare with the corresponding results obtained with higher grid resolutions and more elaborate Riemann solvers (Ryu \& Jones 1995; Jiang \& Wu 1999). Postshock oscillations, which are always produced in any shock-capturing scheme (Arora \& Roe 1996), appear here with vanishing amplitudes behind the fast-moving shocks but have significant sizes near the slow shocks and near the expansion wave on the right-hand side of Figure 3. At present, to our knowledge, no general cure has been envisaged to suppress entirely this unphysical wave noise, which can then be reduced only by adding numerical viscosity. In this sense, the observed oscillations allow to estimate the implicit numerical viscosity of our CENO-LF 


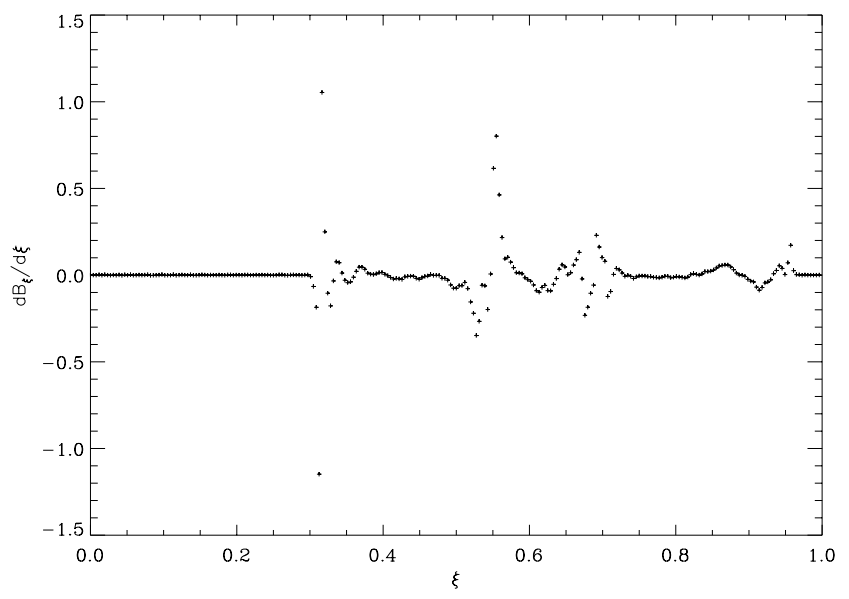

FIG. $6 a$

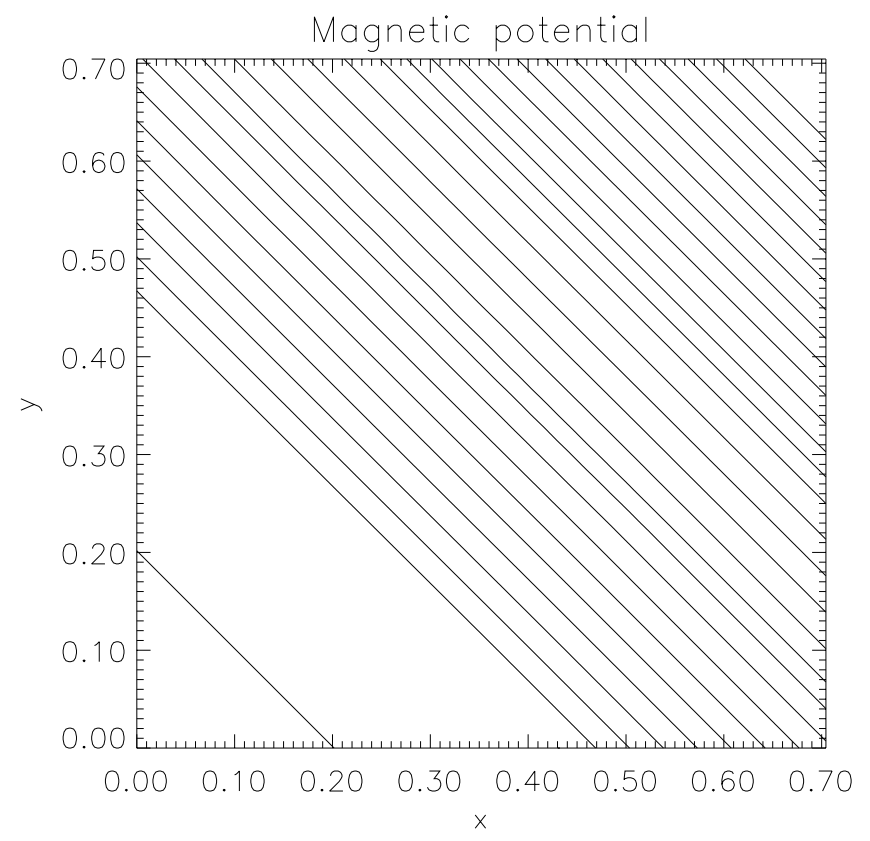

FIG. $6 b$

FIG. 6.-(a) $\xi$ derivative of the $B_{\xi}$ field in the 2D shock-tube problem of Fig. 5. (b) Corresponding isocontours of the magnetic potential $A$, which clearly show the $\eta$-invariance.

scheme to be somehow intermediate between the lower order TVD code of Ryu \& Jones (1995) and the Weno-LLF MHD code of Jiang \& Wu (1999). Other limiters have also been tested (van Leer, SUPERBEE, etc.), with no significant improvements. In any case, we want to stress again the point that high-order schemes not based on characteristics decomposition, like our code, are not particularly designed to handle Riemann problems, in which a lower order scheme may be a better choice.

To test the code for 2D cases, we have run the previous Riemann problems RJ1 and RJ2 with structures propagating along the main diagonal of a computational box with sizes $L_{x}=\cos \alpha, L_{y}=\sin \alpha$, where $\alpha=\pi / 4$. In this way the diagonal has a unit size $L=1$, and $h=1 / N_{x}$ is the size of the cell diagonals. Initial conditions are then assigned to the state vector $\boldsymbol{w}(\xi)=\left[\rho, v_{\xi}, v_{\eta}, v_{z}, B_{\eta}, B_{z}, p\right]^{T}$ along the coor-
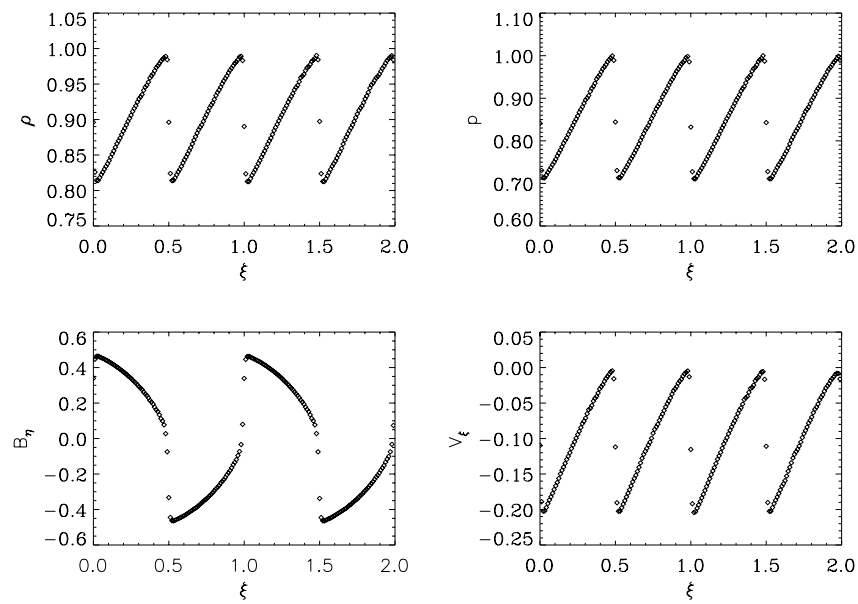

FIG. 7.- Indicated variables for the 2D slow wave problem, along the $\xi$ coordinate and at time $t=1$. The slope angle is $\alpha=\pi / 6$, and the computational box has $192 \times 192$ grid points. dinate $\xi=x \cos \alpha+y \sin \alpha$, with $B_{\xi}=B_{0 \xi}$ now being constant. Boundary conditions are specified by imposing the continuity of all variables along the traverse direction $\eta=y \cos \alpha-x \sin \alpha$, extended to the $x<0, x>L_{x}$ and to the $y<0, y>L_{y}$ sides. We used $N_{x}=N_{y}=256$ grid points, and we found that this grid spacing is hardly sufficient to recover the main flow structures.

The evolved variables $\boldsymbol{w}(\xi)$ are shown in Figure 4 for the rotated 1D Riemann problem of Figure 1 and in Figure 5 for the rotated 1D Riemann problem of Figure 2, at corresponding times. The plotted results are comparable to the ones presented by Ryu et al. (1998) for the same Riemann problems. The fact that the small oscillations observed in the corresponding 1D cases are now less apparent is due to the higher numerical dissipation produced by the lower resolution.

In a $2 \mathrm{D}$ shock-tube problem, the divergence-free condition can be simply expressed by a constant $B_{\xi}$ field, i.e., by the $d B_{\xi} / d \xi=0$ relation along both the $\xi$ and $\eta$ coordinates. However, if $B_{\xi}$ is constructed using the cell centered $\left(B_{x}, B_{y}\right)_{j, k}$ fields, this conservation law is poorly verified, as can be seen in Figure $6 a$, where the numerical derivative $\Delta_{\xi} B_{\xi} / h$ for the RJ2 test is plotted. On the other hand, using the vector potential, point values of the $B_{\xi}$ field are properly defined by

$$
B_{\xi}=B_{0 \xi}+\frac{\partial A}{\partial \eta}
$$

and the divergence-free condition results if $A$ (as well as the other dynamical variables) do not depend on the $\eta$ coordinate. This is documented by the $2 \mathrm{D}$ structure shown in Figure $6 b$. The corresponding numerical derivative

$$
\frac{1}{h} \Delta_{\xi} B_{\xi}=\frac{\Delta_{\xi} \Delta_{\eta} A}{h^{2}}
$$

has now a maximum size of $\simeq 10^{-5}$. 

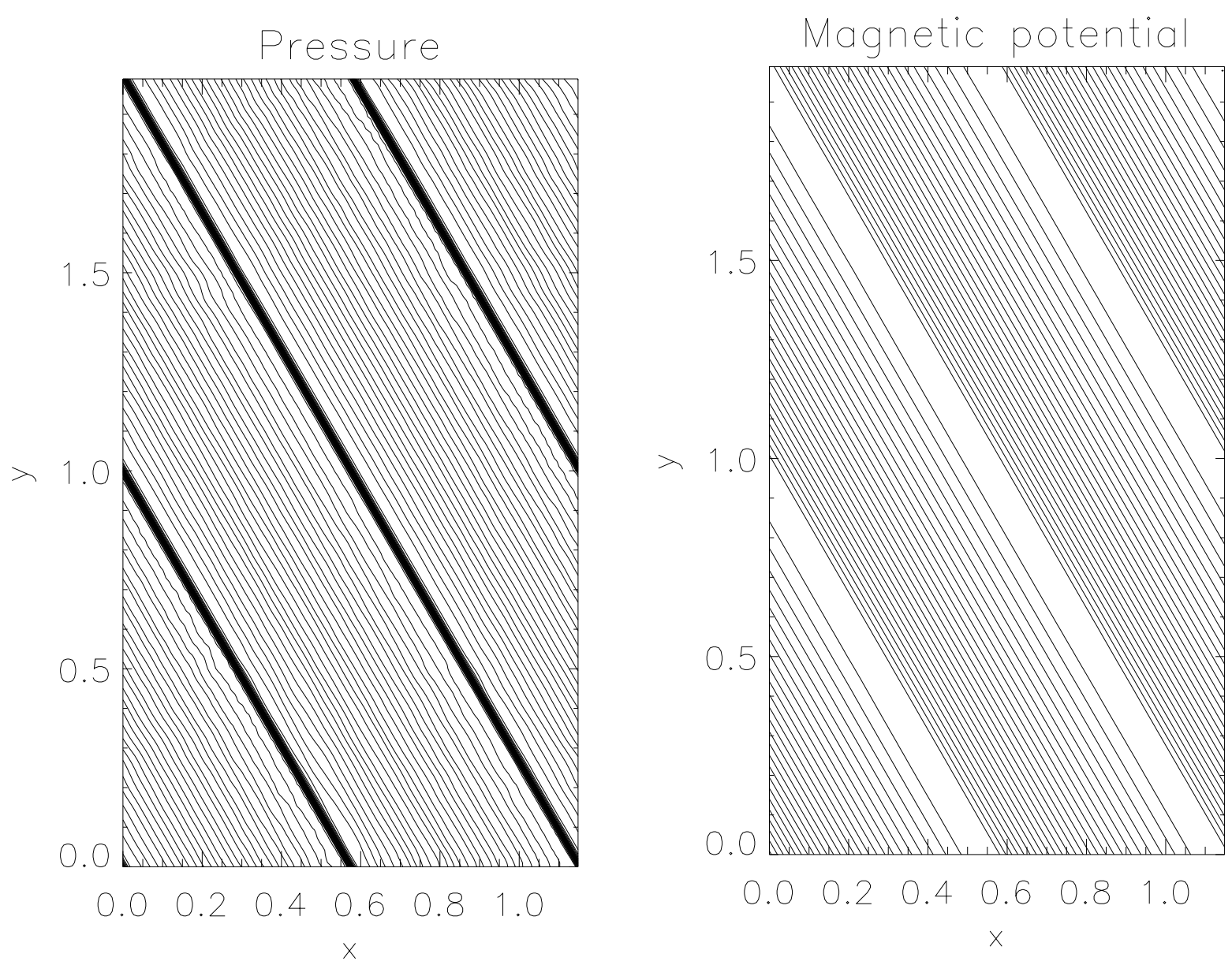

FIG. 8.-(Left panel) Pressure and (right panel) magnetic potential isocontours for the slow wave problem of Fig. 7 at the same time $t=1$

\subsection{Slow Wave Steepening and Shock Formation}

Nonlinear wave steepening from continuous initial data is a main feature of compressible flows. In the MHD case this problem has also interesting astrophysical aspects for the study of intermediate shocks (shocks coupled to expansion waves), already encountered in the previous shock-tube test RJ3. On the computational side, wave steepening is

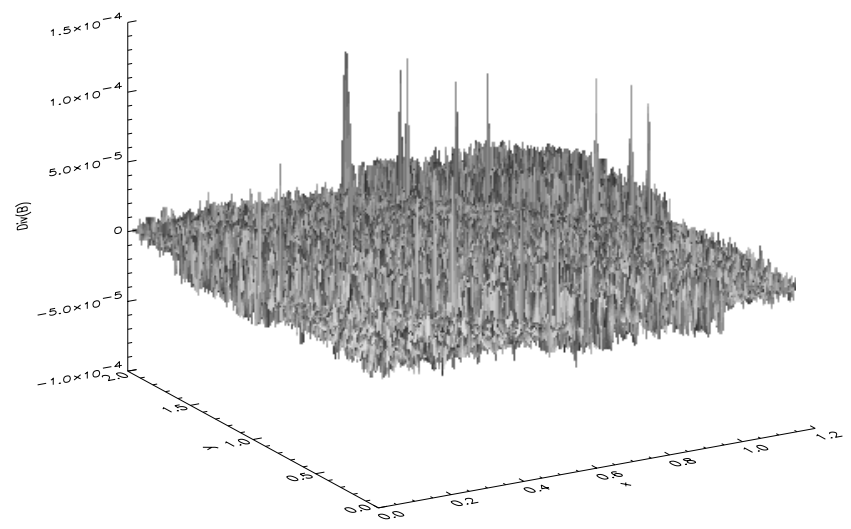

FIG. $9 a$ significant for high-order schemes, whose small numerical dissipation may model weakly resistive plasmas. Reference results are given by $\mathrm{Wu}$ (1987) for physical setting in resistive MHD and by Dai \& Woodward (1998) and Jiang $\& \mathrm{Wu}(1999)$ for numerical testing.

Here we consider initial data defined by a (smooth) slow wave front propagating along the transverse $\xi$ axis, with a

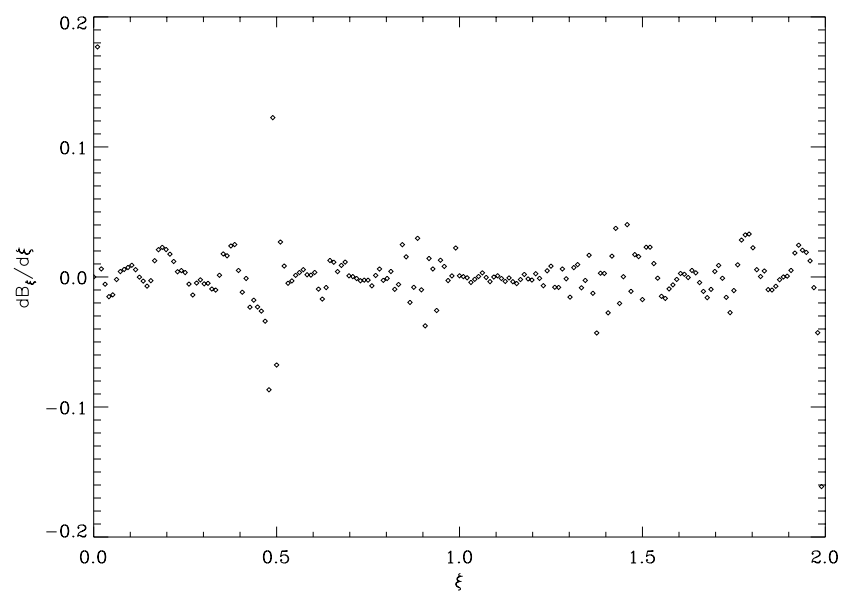

Fig. $9 b$

FIG. 9. - (a) Surface plot of $\boldsymbol{\nabla} \cdot \boldsymbol{B}$ for the slow wave problem at time $t=1$. (b) Numerical derivative of the $B_{\xi}$ field, computed with cell centered $\left(B_{x}, B_{y}\right)$ data. 

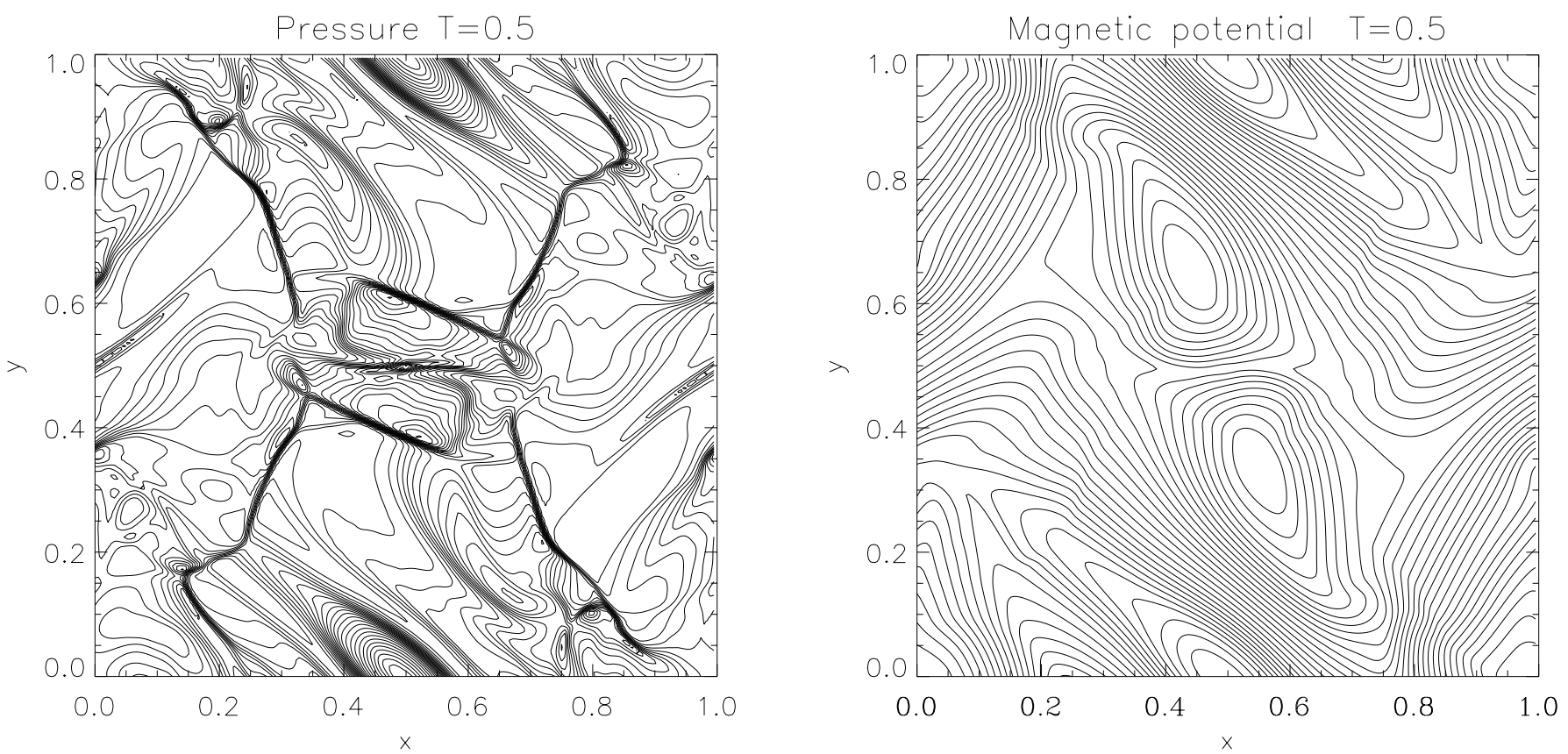

Fig. 10.-(Left panel) Pressure and (right panel) magnetic potential $A$ distribution in the Orszag-Tang problem at time $t=0.5$. The unit square computational box has $192 \times 192$ grid points.

slope angle $\alpha=\pi / 6$ with respect to the $x$ axis. The initial conditions are defined by the characteristic differential equations (the prime here denoting a $\xi$ derivative)

$$
\rho^{\prime}=-\frac{B_{\eta} B_{\eta}^{\prime}}{\left(a^{2}-c_{s}^{2}\right)}, \quad p^{\prime}=a^{2} \rho^{\prime}, \quad q_{\xi}^{\prime}=\frac{c_{s}}{\rho} \rho^{\prime}, \quad q_{\eta}^{\prime}=\frac{c_{f}}{a \sqrt{\rho}} B_{\eta}^{\prime},
$$

relating $\left(\rho, p, q_{\xi}, q_{\eta}\right)$ to the $B_{\eta}$ field along the $\xi$ coordinate. The variables $a, c_{s}, c_{f}$ denote the sound, slow, and fast wave speeds, respectively. We choose as the initial profile $B_{\eta}(\xi)=$ $\sin (2 \pi \xi)$ and a Cartesian box with $L_{x}=1 / \cos \alpha$ and $L_{y}=$ $1 / \sin \alpha$, so that periodic boundary conditions can be applied along the $x$ and $y$ Cartesian coordinates.

In Figure 7 the $\xi$ profiles of the variables $\left(\rho, p, B_{\eta}, v_{\xi}\right)$ are shown at time $t=1$, when a shock train is already formed.
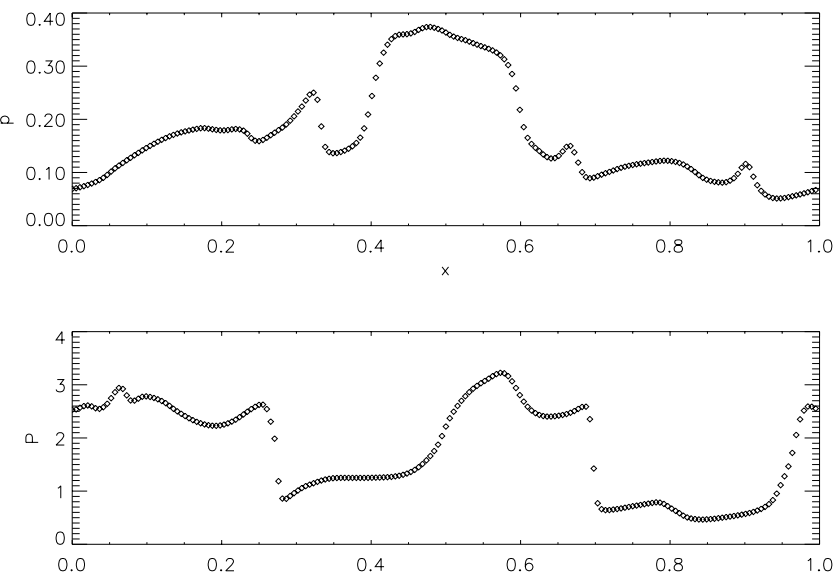

FIG. 11.-1D pressure distribution for the same problem as in Fig. 10 along a cut (upper panel) at $y=0.4277$ and (lower panel) at $y=0.3125$ (where a proper normalized pressure is shown, to compare with the Jiang \& Wu 1999 data).
The corresponding 2D plots of the pressure $p(x, y)$ and of the vector potential $A(x, y)$ are also shown in the Figure 8, to check for accurate $\eta$ independence of the flow variables. As for the previous shock-tube test of Figure 6, a vanishing numerical $\boldsymbol{\nabla} \cdot \boldsymbol{B}$ comes from the $\partial_{\eta} A \simeq 0$ condition. In Figure $9 a$ a surface plot of the variable $D_{B}$ is also shown, giving a value $\left|D_{B}\right|_{\max } \simeq 10^{-4}$ for the residual numerical monopoles, while the $B_{\xi}$ component based on $\left(B_{x}, B_{y}\right)_{j, k}$ values shows a much higher derivative $O\left(10^{-1}\right)$, as can be seen in Figure $9 b$.

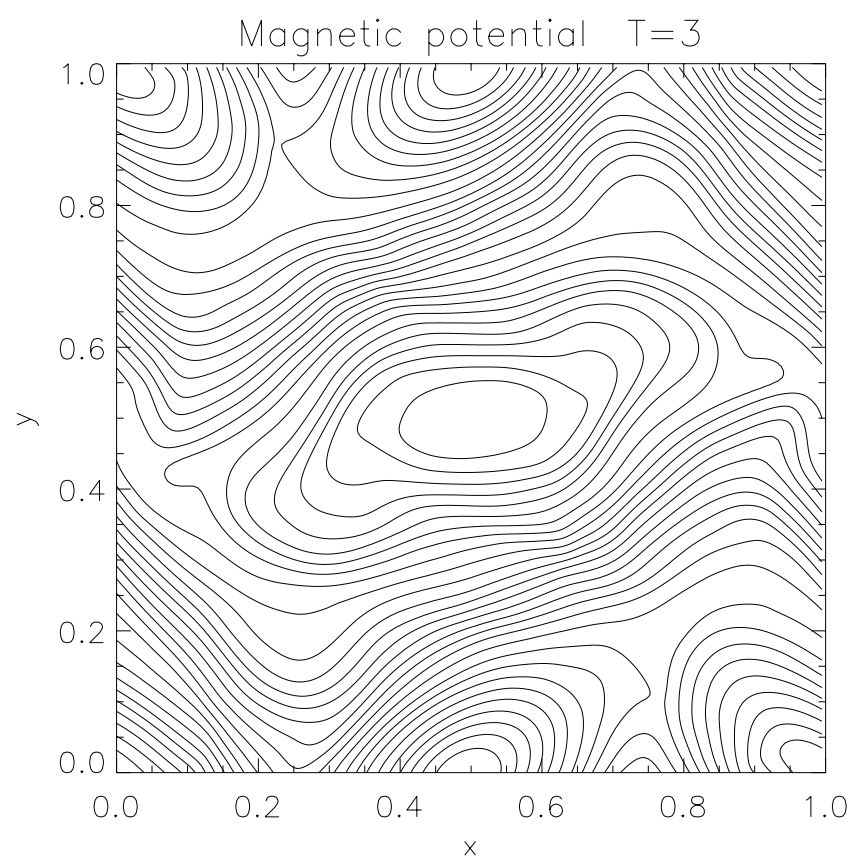

Fig. 12.-Magnetic potential $A$ distribution for the same problem as in Fig. 10 at a later time $t=3$. The reconnected central magnetic island is clearly shown. 

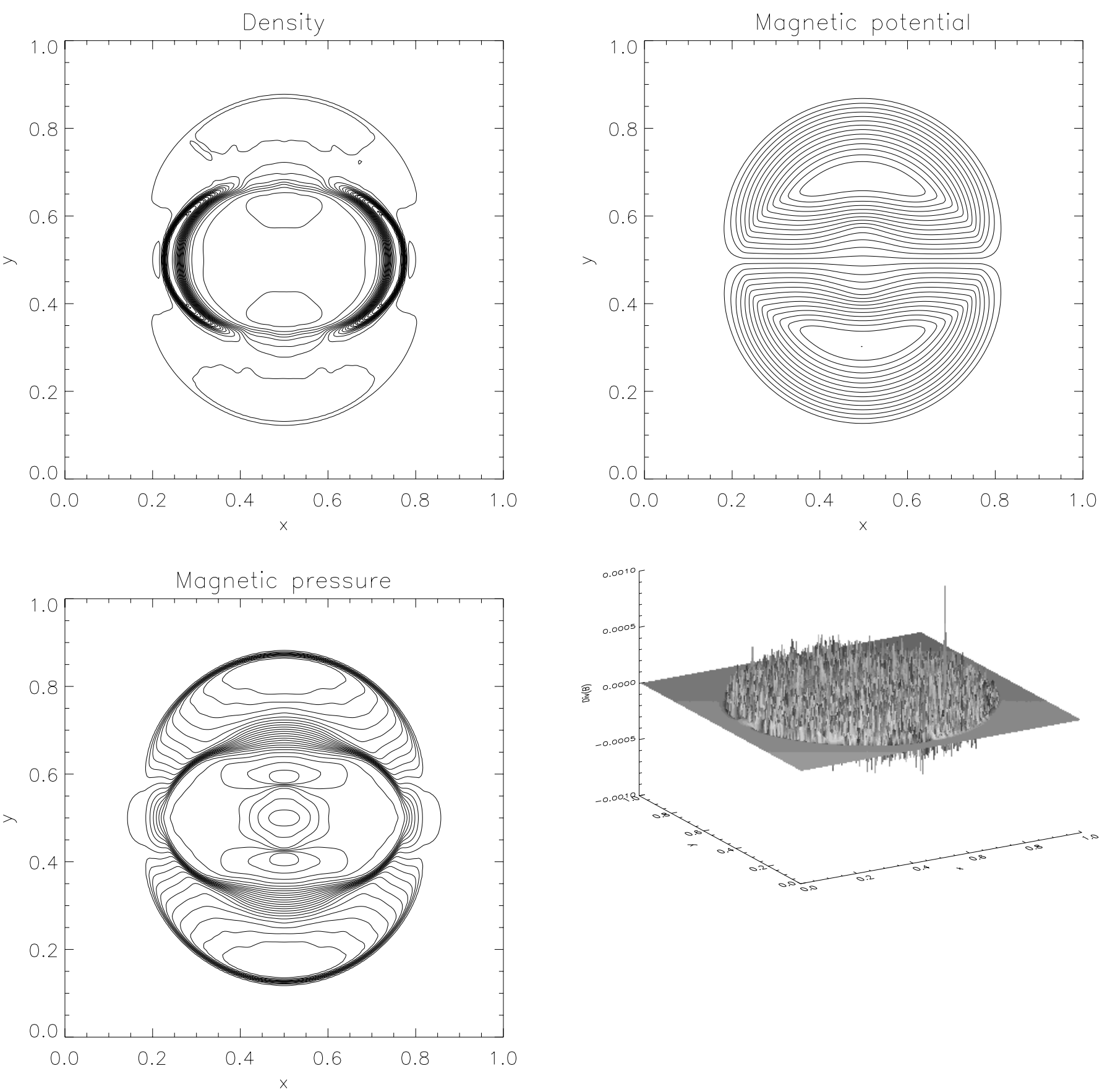

FIG. 13.-Indicated variables at time $t=0.02$ for the blast wave problem. A unit computational box is used with $192 \times 192$ grid points.

\subsection{The Orszag-Tang MHD Vortex Problem}

A well-known model problem to study the transition to MHD turbulence is provided by the so-called Orszag-Tang vortex, which has been extensively studied in its compressible version (for low Mach numbers) by many authors using spectral methods. For initial Mach numbers $M \geq 1$ this is also a valuable test for upwind codes, and it has been used for almost all the latest schemes (Zachary et al. 1994; Dai \& Woodward 1998; Ryu et al. 1998; Jiang \& Wu 1999). The referenced Orszag-Tang system is defined by the initial conditions

$$
\begin{gathered}
v_{x}=B_{x} / B_{0}=-\sin 2 \pi y, \quad v_{y}=\sin 2 \pi x, \\
B_{y}=B_{0} \sin 4 \pi x, \quad p=(\beta / 2) B_{0}^{2}, \quad \rho=\gamma p,
\end{gathered}
$$

where $B_{0}=(4 \pi)^{-1 / 2}$ and $\beta=2 \gamma$ for the usual $\gamma=5 / 3$ value. The initial flow is the given by a velocity vortex superimposed to a magnetic vortex, with a common (singular) $X$-point, but with a different modal structure. This configuration is strongly unstable, giving rise to a wide spectrum of propagating MHD modes and shock waves (here the initial Mach number is $M=1$ ) and to the transformation of the initial $X$-point to a current-sheet triggering the reconnection process.

For this test we have chosen a unit grid $L_{x}=L_{y}=1$ with $N_{x}=N_{y}=192$ collocation points. In Figure 10 we present the pressure $p$ and potential $A$ isocontours at $t=0.5$, showing the good qualitative agreement with the other published works. In particular, 1D profiles of the $p(x)$ variable (upper panel) at $y=0.4277$ and (lower panel) at 

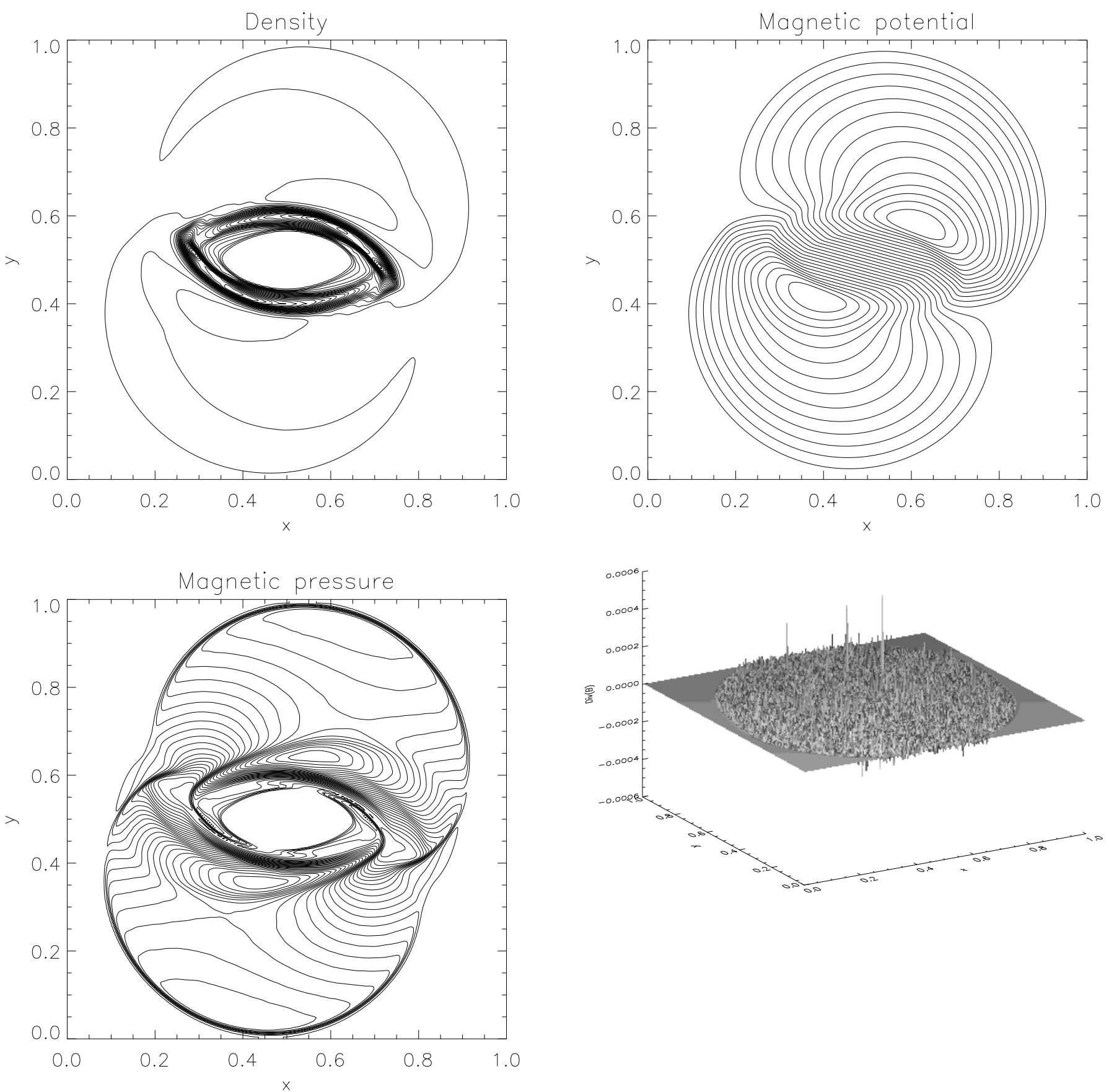

FIG. 14.-Indicated variables at time $t=0.18$ in the rotor problem. A unit computational box with $240 \times 240$ grid points is used.

$y=0.3125$ are shown in Figure 11 for a more detailed comparison with the corresponding plots given, respectively, by Ryu et al. (1998) and Jiang \& Wu (1999). The latter reference also contains some quantitative estimate of how significant magnetic monopoles may affect the computed solutions, thus producing numerical instabilities in a long time evolution.

The monopole distribution $D_{B}$ (not shown) show only a few enhanced values $\simeq 10^{-5}$, thus assuring vanishing $\nabla \cdot B$ condition. in the long time computations we found no evidence of negative pressure nor other unphysical behaviors. To check this point in more detail, we plot the magnetic field lines at $t=3$ in Figure 12, showing how the regularity of field lines is well preserved in time. This essential feature of the computed divergence-free magnetic field allows to reproduce typical magnetic phenomena, like the topology change induced by a vanishing resistivity (here modeled by a low numerical diffusivity). In fact, by comparing the latter $A$ distribution with the former shown in Figure 10, one sees how the initial magnetic islands around the $X$-point merge by reconnection.

\subsection{Strong Blast Wave in Free Space}

The following two numerical tests concern the formation and propagation of strong MHD discontinuities in a 2D domain. These model problems are representative of many astrophysical phenomena in which the magnetic energy has relevant dynamical effects. In numerical schemes having 
poor divergence-free properties, the (possible) onset of spurious solutions and of a negative gas pressure is clearly enhanced in these physical regimes, since the magnitude of numerical monopoles increases along with the background magnetic pressure. This problem has been discussed, in particular, by Balsara \& Spicer (1999), where some quantitative estimate of the numerical $\boldsymbol{\nabla} \cdot \boldsymbol{B}$ produced by Godunov schemes has also been documented.

The first test problem concerns the explosion of a circular dense cloud in a magnetized, initially static region. Here we take again a square domain with $N_{x}=N_{y}=192$ grid points. Initial conditions are specified by filling a circular region located at the center and radius $r_{0}=0.125$ with a hot gas having $p=100$. The background static fluid is characterized by $\rho=p=1$ and $B_{0 x}=10$.

In Figure 13 we show the density $\rho$, the magnetic potential $A$, the magnetic pressure $\left(B_{x}^{2}+B_{y}^{2}\right) / 2$, and the solenoidal variable $D_{B}$ distributions at time $t=0.02$, which is already representative of the generated complex flow structure. In particular, the plotted results show the well-preserved initial axial symmetries (around both the $y=0.5$ and the $x=0.5$ axis) as well as the regularity of magnetic field lines. As we can see, the resulting numerical $D_{B}$ variable has an isolated peak with magnitude $10^{-3}$ and otherwise vanishing sizes $<10^{-5}$.

\subsection{The Fast Rotor Problem}

In Balsara \& Spicer (1999), a model problem to study the onset and propagation of strong torsional Alfvén waves, relevant for star formation, was presented and analyzed. Following this reference, we have run the same problem using a square unit computational box and $N_{x}=N_{y}=240$ grid points. For propagating structures not intersecting the box boundaries, periodic conditions can be applied. Initial conditions are specified by a rapidly rotating cylinder (the rotor) with center at the $x=0.5, y=0.5$ point and radius $r=0.1$. The rotor has (initial) density $\rho=10$ and angular velocity $\omega=20$, and it is embedded in a static and uniform fluid with $\rho=p=1$ and $B_{0 x}=2.5 \pi^{-1 / 2}$.

The evolved flow pattern at time $t=0.18$ (just before the shocked flow reaches the boundaries) is shown in Figure 14, representing as in Figure 13 the space distribution of the density $\rho$, the magnetic potential $A$, the magnetic pressure $\left(B_{x}^{2}+B_{y}^{2}\right) / 2$, and the solenoidal variable $D_{B}$, which remains below $4 \times 10^{-4}$ everywhere. Even if a lower resolution than in the referenced paper is adopted here, and no smoothing is applied to the initial density and rotation velocity discontinuities, the numerical results give convincing evidence about how a higher order scheme provides accurate and well-resolved profiles even when strong discontinuities develop.

\section{CONCLUSIONS}

We have introduced a general method to adapt upwind schemes developed for Euler system to the corresponding MHD system in order to assure the divergence-free condition. The proposed approach can be applied to existing MHD codes as well as to any higher order extensions.

The use of a staggered collocation for the magnetic field components entering the $\boldsymbol{\nabla} \cdot \boldsymbol{B}$ variable and of the related magnetic potential $A$ are well-known general premises to represent a numerical divergence-free magnetic field at the second-order accuracy level. We have thus introduced proper algorithms to extend this representation to higher orders and to formulate upwind flux derivatives using only the divergence-free variables, in order to avoid the onset of numerical monopoles in the momentum and energy equations. Moreover, by taking into account consistency arguments, we have proposed a new formulation for the upwind flux for the induction equations.

As an application, we have constructed a simple and efficient third-order LF-CENO based MHD code running in multidimensional systems. This code appears to be well suited for many astrophysical problems in which, beside strong shocks, reconnection phenomena, complex wave patterns, and turbulence develop, as confirmed by the several numerical tests presented here.

The authors would like to thank Marco Velli for many helpful discussions and for his support in completing this work.
Arora, M., \& Roe, P. L. 1996, J. Comput. Phys., 130, 25

Balsara, D. S. 1998a, ApJS, 116, 119

.1998b, ApJS, 116, 133

Balsara, D. S., \& Spicer, D. S. 1999, J. Comput. Phys., 149, 270 (BS)

Barmin, A. A., Kulikovskiy, A. G., \& Pogorelov, N. V. 1996, J. Comput. Phys., 126, 77

Brackbill, J. U., \& Barnes, D. C. 1980, J. Comput. Phys., 35, 426

Brio, M., \& Wu, C. C. 1988, J. Comput. Phys., 75, 400

Cargo, P., \& Gallice, G. 1997, J. Comput. Phys., 136, 446

Chen, G.-Q., \& Lefloch, P. G. 1995, Commun. Pure Appl. Math, 48, 691

Dai, W., \& Woodward, P. R. 1998, ApJ, 494, 317 (DW)

DeVore, C. R. 1991, J. Comput. Phys., 92, 142

Evans, C., \& Hawley, J. F. 1988, ApJ, 332, 659

Harten, A. 1983, J. Comput. Phys., 49, 357

Harten, A., Engquist, B., Osher, S., \& Chakravarthy, S. 1987, J. Comput. Phys., 71, 231

Harten, A., Lax, P. D., Levermore, C. D., \& Morokoff, W. J. 1998, SIAM J. Numer. Anal., 35, 2117

\section{REFERENCES}

Jiang, G.-S., \& Wu, C.-C. 1999, J. Comput. Phys., 150, 561

Jin, S., \& Xin, Z. 1998, SIAM J. Numer. Anal., 35, 2385

Liu, X.-D., \& Osher, S. 1998, J. Comput. Phys., 142, 304

Powell, K. G. 1994, Instit. Comput. Appl. Sci. Eng. Rep. 94-24

Roe, P. L., \& Balsara, D. S. 1996, SIAM J. Numerical Anal., 56, 57

Ryu, D., \& Jones, T. W. 1995, ApJ, 442, 228

Ryu, D., Jones, T. W., \& Frank, A. 1995, ApJ, 452, 785

Ryu, D., Miniati, F., Jones, T. W., \& Frank, A. 1998, ApJ, 504, 244 (RY)

Shu, C.-W. 1997, Instit. Comput. Appl. Sci. Eng. Rep. 97-65, Langley, VA

Shu, C.-W., \& Osher, S. 1988, J. Comput. Phys., 77, 439

. 1989, J. Comput. Phys., 83, 32

Stone, J. M., \& Norman, M. L. 1992, ApJS, 80, 791

Tadmor, E. 1988, Instit. Comput. Appl. Sci. Eng. Rep. 88-41

van Leer, B. 1979, J. Comput. Phys., 32, 101

. 1982, in Lecture Notes Phys., 170, 507

Wu, C.-C. 1987, Geophys. Res. Lett., 14,668

Zachary, A. L., Malagoli, A., \& Colella, P. 1994, SIAM J. Sci. Stat. Comput., 15, 263 